\title{
59. PRELIMINARY SEDIMENTOLOGY OF LATE QUATERNARY DIATOMACEOUS MUDS FROM DEEP SEA DRILLING PROJECT SITE 480, GUAYMAS BASIN SLOPE, GULF OF CALIFORNIA ${ }^{1}$
}

\author{
Kerry Kelts, Eidgenössische Technische Hochschule, Geologisches Institut, Zürich, Switzerland \\ and \\ Jeffrey Niemitz, Department of Geology, Dickinson College, Carlisle, Pennsylvania
}

\begin{abstract}
This paper provides a brief, descriptive, sedimentological background for the chapters on hydraulic piston core Site 480 in this symposium, and supplements data given in the site chapter for Sites $479-480$ (this volume, Pt. 1). Sediments are composed primarily of planktonic diatoms, with minor numbers of silicoflagellates, radiolarians, and varying amounts of both benthic and planktonic foraminifers, along with a large terrigenous component of olive brown, silty clay. The section contains meter-thick intervals of finely laminated facies alternating with nonlaminated zones. A few paleoenvironmental events are documented within the generally uniform sequence by sporadic occurrences of thin turbidites, phosphatic concretions, fish debris concentrations, an ash layer, and a thin layer of diagenetic dolomite. The distribution of nonlaminated and laminated zones is attributed to fluctuations of bottom-water oxygen content caused by variations in circulation, fertility, and productivity. Homogeneous sections are interpreted as coinciding with cooler climatic periods, whereas laminated sections seem to correspond to upwelling conditions during drier periods.
\end{abstract}

\section{INTRODUCTION}

Interest has focused on the Gulf of California, not only as the site of oblique rifting and the formation of young ocean basins, but also as a major silica sink in the form of massive diatom productivity. A girdle of laminated diatom ooze around the Guaymas Basin Slope was demonstrated for the uppermost meter of sediment by the first E.W. Scripps cruise in 1939 (Revelle, 1950). The mechanism of formation of the light and dark couplets and their relation to changing oceanographic parameters is a topic of continuing debate.

Revelle's (1950) observations were followed by those of Byrne and Emery (1960), who interpreted the couplets as varves formed by an annual diatom bloom overprinting constant terrigenous input. Calvert $(1964,1966)$ studied a series of short cores to define the spacial distribution of laminated and homogeneous facies. His conclusions diverged from previous models by ascribing prime variability to the terrigenous input, overprinting a moderately fluctuating diatom assemblage. Calvert (1966) had shown that fluctuations of the $\mathrm{O}_{2}$-minimum are imprinted on a regional pattern of sedimentation. By the time of the Leg 64 cruise it was clear that the mechanism of varve formation was more complex, possibly diachronous, and involved the interaction of seasonal deposition of terrigenous sediment and deposition of products of upwelling and of oceanic biogenous phases (Fig. 1).

Rhythmically laminated marine sediments are rare. They occur in areas of seasonally differentiated sediment flux, where bottom waters are sufficiently oxygendepleted to prevent an infauna from churning the seasonal signal (e.g., Soutar and Crill, 1977). Such sedi-

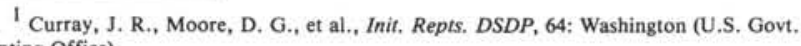
Printing Office). ments provide a high-resolution record of paleoceanographic variation, particularly rate of change, and offer an opportunity for correlation of the land-sea climate record in regions such as the Gulf of California.

DSDP Leg 64 tested the hydraulic piston corer (HPC) at Site 480 (water depth $655 \mathrm{~m}$ ) in order to (1) determine what vertical variations in the pattern of sedimentation at a single location had occurred during the late Pleistocene; and (2) test whether these could be related to shifts in circulation patterns coupled with climate and sealevel change. The HPC provided undisturbed samples from the surface to 152 meters sub-bottom that were infinitely superior to equivalent cores from Site 479, drilled only $6 \mathrm{~km}$ away with conventional rotary techniques (see Fig. 2). The sediment record undoubtedly needs intensive further research for its potential as a reference section for the late Pleistocene, and this description is intended only as a guide to its quality, extent, and limitations. The description is based on macroscopic observations of sediment structure, supplemented by mineralogical and microtexture data. Figure 3 gives a synopsis of the stratigraphic position and recovery of the hydraulic piston cores and includes comments on sedimentary features observed while we were shaving off $10-\mathrm{cm}$ intervals of the core surface to provide integrated samples for pollen and oxygen isotope analysis (Heusser, this volume, Pt. 2; Shackleton, this volume, Pt. 2). These features were not included in site chapter descriptions because shaving and sampling of the Hole $480 \mathrm{HPC}$ cores was deferred until radiographs could be made of the undisturbed sections (Soutar et al., this volume, Pt. 2).

\section{SEDIMENTS}

The characteristic feature of the sequence in Hole 480 is the existence of two alternating sediment types, distinguished by primary sedimentary structures. The composite section (Fig. 4) is divided almost equally between 

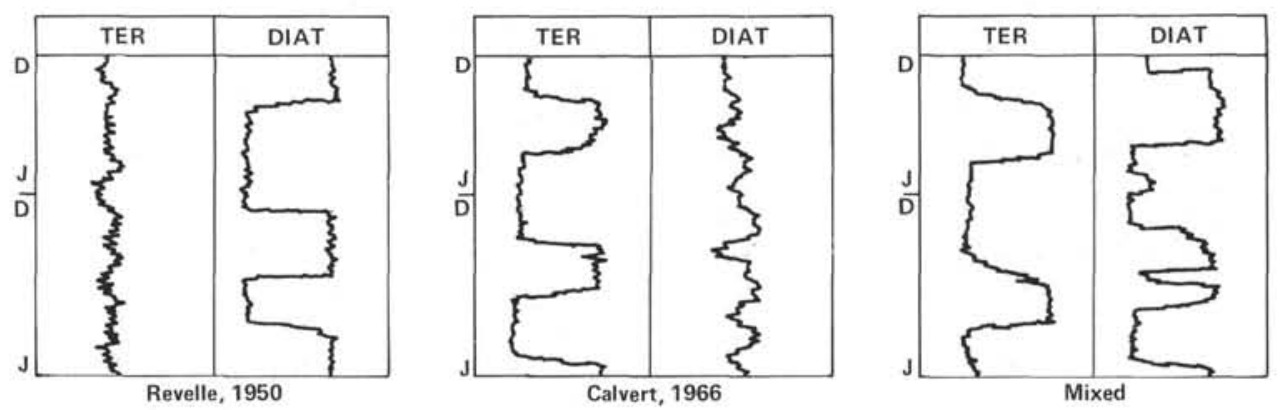

Figure 1. Three contrasting hypothetical models for the input of a seasonal signal to produce light and dark laminae in the diatom-rich hemipelagic oozes of the Guaymas Basin, Gulf of California. ( $\mathrm{J}=\mathrm{January}$ to $\mathrm{D}=$ December; $\mathrm{TER}=$ terrigenous; $\mathrm{DIAT}=$ diatomaceous. $)$

(1) zones comprising rhythmically laminated couplets of pale olive diatom ooze and darker, moderate olive brown muddy diatomaceous ooze and, (2) zones of moderate olive to brown homogeneous diatomaceous muds to ooze. In addition, there are sporadic sand layers, graded beds, glauconite pellets, phosphatic concretions, fish debris, an ash layer, and a dolomitic mudstone. The sediments contain signals from both marine productivity (diatoms, radiolarians, nannofossils, benthic and planktonic foraminifers, dinoflagellates, fish scales, and organic carbon) and continental influences (terrigenous clays, silts and sands, pollen, plant debris, and organic carbon). The water content decreases from $85 \%$ in the first core to about $70 \%$ by Core $3(10 \mathrm{~m})$ which suggests that the top was relatively near the sediment/ water interface. Cores below the level of Core $19(90 \mathrm{~m})$ are flaky and crumble if thin slices are cut because of abundant fibrous diatom frustules (Chaetoceros bristles and Thalassionema and Thalassiothrix species). These zones may also have had significant amounts of biogenic gas within pore spaces.

Revelle (1950) described several salient features we also encountered in Hole 480, including the abundance of $\mathrm{H}_{2} \mathrm{~S}$ and methane as well as zones of laminated (0.1$2 \mathrm{~mm}$ ) and nonlaminated muds. He interpreted gray muds which interrupt laminated sequences as a deposit from sheet floods originating from the Yaqui River. Some glauconite was found in outer shelf areas. Yellowish layers he ascribed to bentonite may in fact be calcareous laminae similar to the dolomite layers in Hole 480 . Contrasting features of laminated versus nonlaminated sediment types are shown in Fig. 5.

A survey of bulk mineralogical compositions from core-catcher samples is given in Table 1 . These show a characteristic hemipelagic development with relatively modest amounts of clay minerals. The spectrum is dominated by quartz and a mixture of various feldspars including abundant plagioclase. Carbonates are variable. From these X-ray data, the first traces of presumably diagenetic dolomite are already present in Core 480-10, or only 45 meters sub-bottom. The dolomite occurs together with stoichiometric calcite. Traces of Mg-calcite are present in several samples down to Core 480-30. Traces of possible early zeolite formation (clinoptilolite) were noted in conjunction with sandy layers (Sample 480-12,CC).

The amorphous component signal (diatom frustules) is ubiquitous but variable and is most prominent in Core $480-18$, which seems to represent a zone that derived from favorable upwelling conditions. The abundance of pyrite follows a similar trend, because framboids are commonly contained within diatom frustules. The clay minerals, which are not quantitatively abundant, have a general illite-chlorite dominance in the upper sections and slightly higher smectite components in the lower zones. In general, peaks are broad, suggesting weathered clastic sources. Some clay mineral peaks (e.g., in Sample 480-30,CC) indicate traces of glauconite. Two samples from a Type II graded mud from Site 481 in the deep Guaymas Basin are included in Table 1 for comparison. Their spectrum, with abundant quartz and chlorite, suggests that these gray basinal muds do not derive from the same hemipelagic sediments that Site 480 comprises and that they may include possibly reworked hydrothermal deposits (see Gieskes et al., this volume, Pt. 2; Einsele and Kelts, this volume, Pt. 2).

\section{Laminated Zones (Fig. 6)}

Rhythmite couplets are mixtures of biogenic and terrigenous components. The pale olive lamina is generally a nearly pure diatom ooze with $70-80 \%$ diatoms and $15-25 \%$ terrigenous clay. The dark lamina is a moderate olive brown, muddy diatomaceous ooze with $45-60 \%$ terrigenous clay and $15-45 \%$ diatom frustules. Some beds have up to $10 \%$ nannofossils (in Cores $3,4,14,15$, and 29) along with variable amounts of other marine microfossils. Sections 480-19-2 and 480-21-3 have layers with between 18 and $40 \%$ nannofossils. Laminae are of variable thicknesses, mostly less than a millimeter; either the light or the dark lamina may be thicker. Variations in lamination thickness and composition commonly occur in packages, giving the core a second-order banded appearance. In some sections where the light olive diatom laminae are almost unrecognizable, dark laminations are well developed, as is shown by X-radiographs (Soutar et al., this volume, Pt. 2). Schrader (this volume, Pt. 2) shows that downhole, either the upwelling or the oceanic diatom assemblages may be dominant 

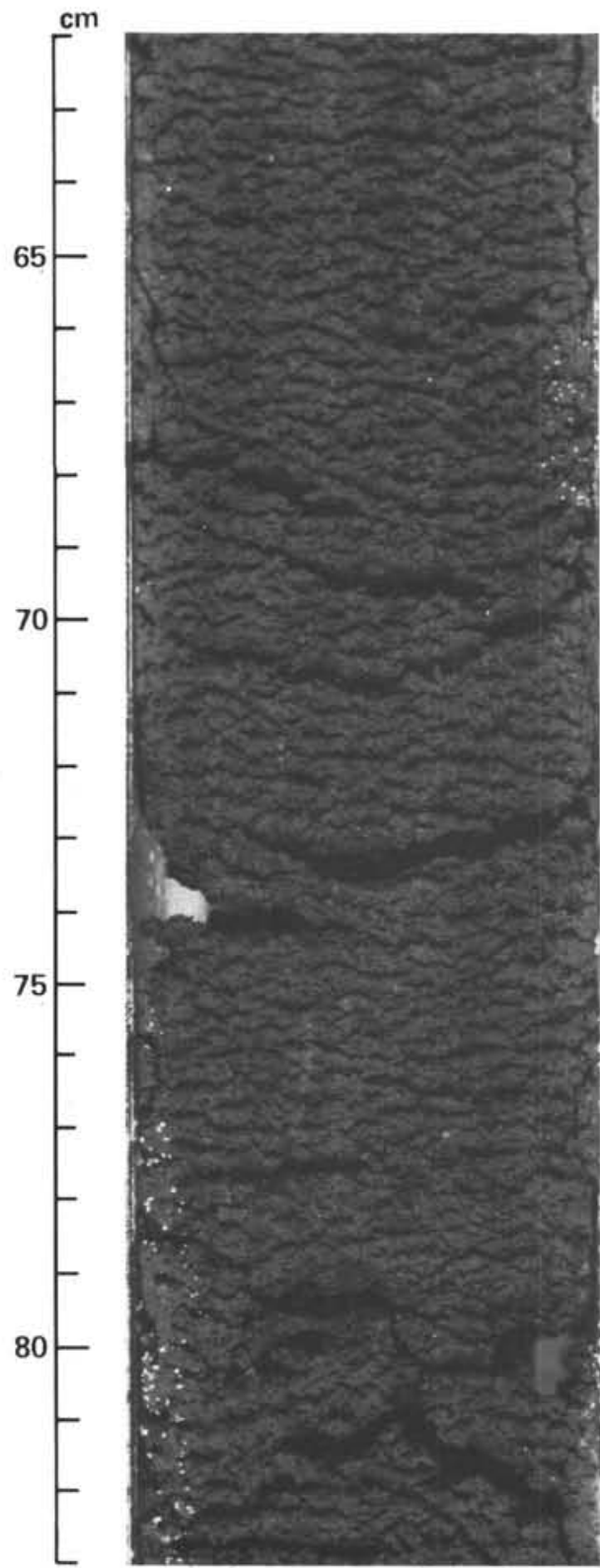

A

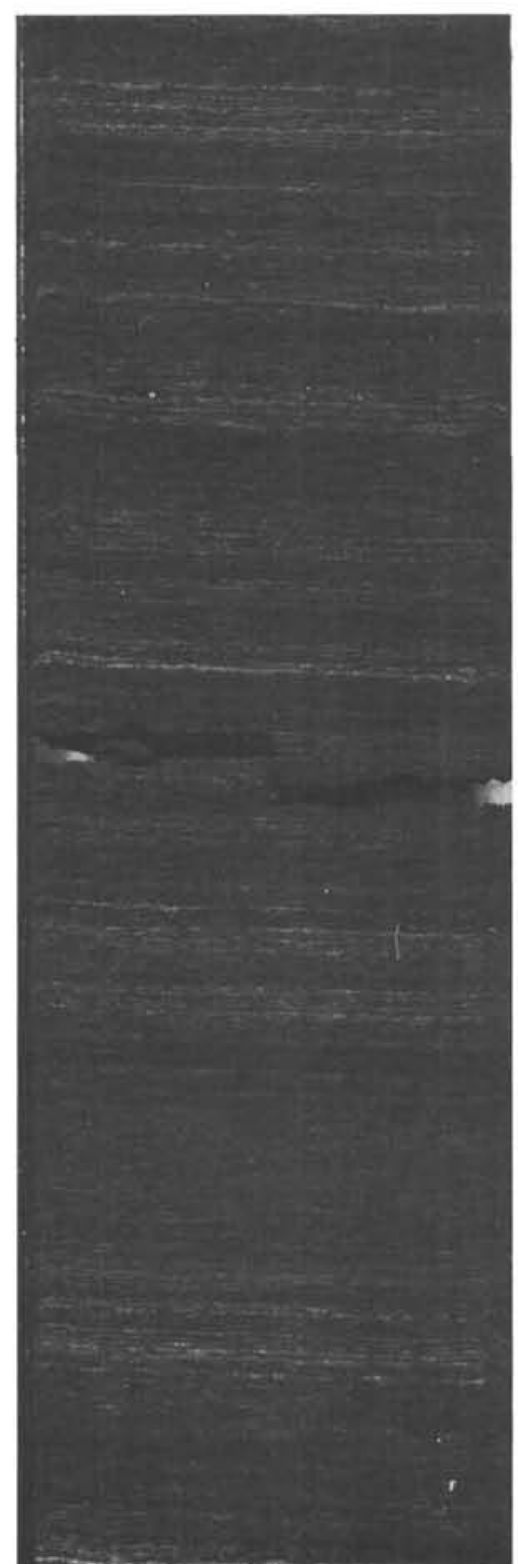

B

Figure 2. Comparison of conventional and hydraulic piston cores from equivalent depths in soft diatomaceous ooze along the Guaymas Basin Slope. A. Disturbed Section 479-12-1 (98-107 m sub-bottom) shows uniform, moderate olive brown (5Y 4/4), muddy diatomaceous ooze with a few pale olive $(106 / 2)$ streaks. Core surface shows numerous gas pockets and gas exsolution textures. B. HPC Section 480-20-2 (95-99 m sub-bottom) shows rhythmic, mm-scale, varvelike alterations of moderate olive brown (5Y 4/4) muddy diatomaceous ooze and pale olive (10Y $6 / 2$ ) diatom ooze. A gray sand layer abuts discordantly against the varves with sharp contact, but shows no grading.

in the light olive member. Byrne and Emery (1960) noted that the light member commonly sets in abruptly with a sharp basal contact and attenuates upward. The layering is mostly parallel and shows uniform thickness over the width of the core.

\section{Nonlaminated Zones}

Zones without laminations consist of moderate olive gray diatomaceous mud to muddy ooze, very similar to the dark member of the rhythmites. The lack of distinct sedimentary structure is characteristic (Fig. 5) but the sediments also have fewer diatoms (10-40\%) and more abundant calcareous nannofossils and foraminifers (10$15 \%)$ than the bulk laminites. Terrigenous clay (40-60\%) includes quartz (6-10\%) and feldspars, mainly plagioclase $(5-12 \%)$. Pyrite is a ubiquitous minor constituent, commonly appearing as framboids within complete diatom frustules.

Strictly speaking, homogeneous is a misnomer, as many of the nonlaminated zones show evidence of faint 

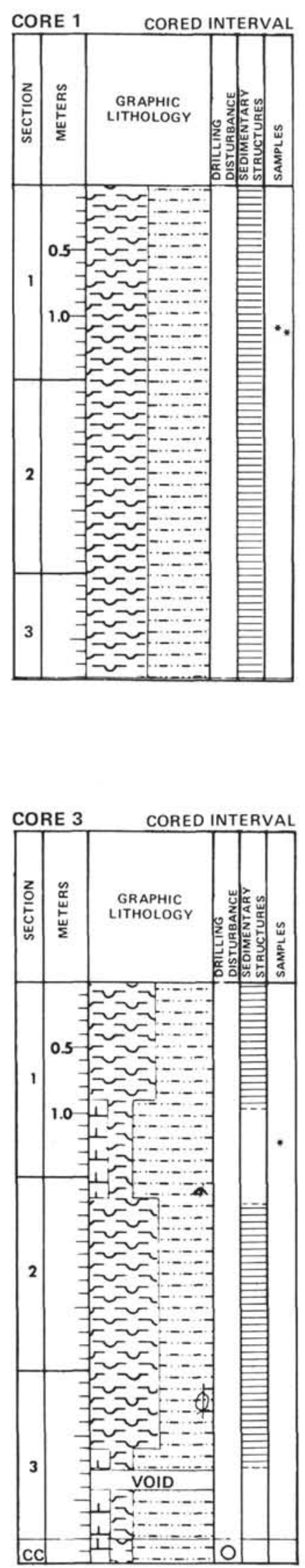

CORE $1 \quad 0.00-4.75 \mathrm{~m}$

Section 1: extremely soft, high water content.

Section 2: reduction of number of feltlike pale olive tufts. Section 2, 143-150 cm: enterolithic fold.

Section 3, $15 \mathrm{~cm}$ : several-mm thick, pale.

Section 3, 62-64 cm: homogeneous.

CORE $2 \quad 4.75-9.50 \mathrm{~m}$

Section 2, 0-30 cm: disturbed but relicts show lamination. Section 2, 40-50 cm: scattered thin homogeneous.

Section 2, 52-53 cm: homogeneous.

Section 2, 80-90 cm: minor deformation, inclined lamination.

Section 2, 120-130 cm: darker, and fine lamination, rare tufts, decline productivity.

Section 3, 36 and $38 \mathrm{~cm}$ : thick pale lamination, trace wood. Section $3,55 \mathrm{~cm}$ : thick pale lamination.

Section $3.80 \mathrm{~cm}$ : thin burrowed layers $0.5 \mathrm{~cm}$.

\section{CORE 3 9.50-14.25 m}

Section 1, 0-10 cm: disturbed, lamination faint.

Section 1, 32-33 cm: homogeneous;

diatomaceous tufts common.

Section 1, $47 \mathrm{~cm}$ : thick diatomaceous ooze.

Section 1,64 cm: thin-shelled mollusk, cellular stone.

Section 1, 92-98 cm: deformation, thin slump. cross lamination.

Section 1, 98-100 cm: trans, banded homogeneous.

Section 1, 107-109 cm: dark band, nannofossil-rich.

Section 2, 20-25 cm: several thick pale diatomaceous ooze, layer.

Section 2, 25-27 cm: homogeneous.

Section 2, 44-46 cm: homogeneous, burrows.

Section 2, 50-60 cm: many thick pale diatomaceous ooze layer.

Section 2,61 cm: rich in organic matter.

Section 2, 67-69 cm: thick diatomaceous ooze.

Section 2, 69-76 cm: rip-up clasts, enterolithic folds, large burrows.

Section 3: light mottles.

Section $3,27 \mathrm{~cm}$ : large wood fragment, banded $\mathrm{cm}$-scale, layered.

Section $3,52 \mathrm{~cm}$ : three thin layers lamination.

Section 3, 60-64 cm: layered foraminifer-rich.

Section 3, 70-80 cm: transition to 73 , diffuse, lamination.

Section 3, 79-81 cm: thick pale diatomaceous ooze, $1 \mathrm{~mm}$.

Section 3, $105 \mathrm{~cm}$ : dark lamination.

Section 3, 111-112 cm: mud layer.

Section $3,113 \mathrm{~cm}$ : thick diatomaceous ooze.

CORE 4 14.25-19.00 m

Section 1, 0-30 cm: disturbed.

Section 1,40 cm: patchy mottles.

Section 1, 75-90 cm: increase in foraminifers.

Section 1, 82-83 cm: rich in foraminifers.

Section 1, 122-139 cm: gray hue, faint fold deformation.

Section 2, $30 \mathrm{~cm}$ : faint mottles.

Section 2, $41 \mathrm{~cm}$ : thin-walled mollusks.

Section $2,89 \mathrm{~cm}$ : thin shell.

Section 2, $110 \mathrm{~cm}$ : more light to dark banding, faint.

Section 3, $58 \mathrm{~cm}$ : mottles with mottled pale diatomaceous ooze, benthics, silty.
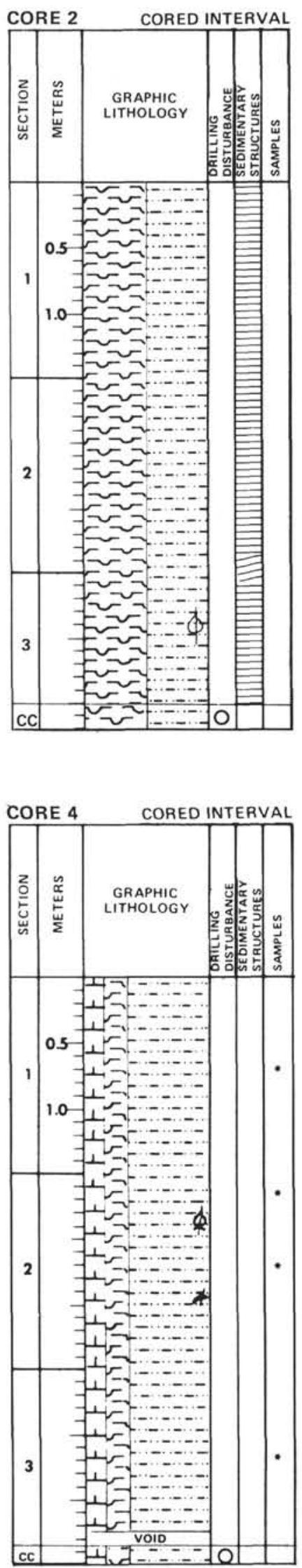

Figure 3. Detailed core lithology as a guide to secondary core features from descriptions of $10-\mathrm{cm}$ scrape intervals, supplementing the site chapter (this volume, Pt. 1). 

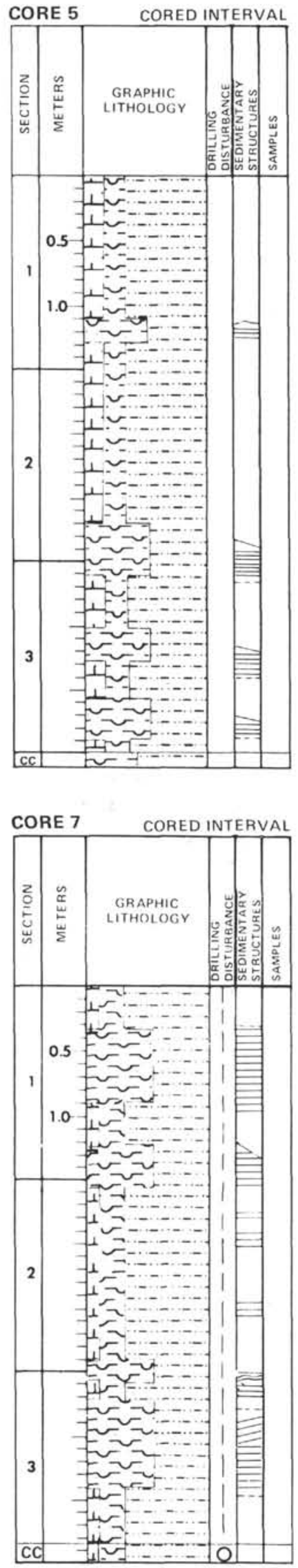

CORE $5 \quad 19.00-23.75 \mathrm{~m}$

Section 1, 0-16 cm: disturbed.

Section 1, $50 \mathrm{~cm}$ : large mottles.

Section 1, $70 \mathrm{~cm}$ : three thin layers.

Section $1,80 \mathrm{~cm}$ : black streaks.

Section 1, 108-121 cm: evidence lamination, but burrowed.

Section 1, $131 \mathrm{~cm}$ : trace lamination.

Section 2, $30 \mathrm{~cm}$ : mottles.

Section 2, 130-137 cm: unconformity.

Section 2, $138 \mathrm{~cm}$ : diffuse layering contact.

Section 3, 0-60 cm: burrows in lamination.

Section 3, 7-10 cm: layering.

Section $3,52 \mathrm{~cm}$ : transition.

Section 3,58-67 cm: layered.

Section 3,98 cm: transition.

CORE $6 \quad 23.75-28.50 \mathrm{~m}$

Section 1, 0-20 cm: disturbed.

Section 1, 33-37 cm: mixed.

Section 1, $37 \mathrm{~cm}$ : lamination.

Section 1, $40 \mathrm{~cm}$ : homogeneous.

Section 1, 45-47 cm: dark band.

Section 2, 10-11 cm: lamination, very fine; burrow mottles.

Section 2, 22-68 cm: large burrow patches.

Section 2, 59-68 cm: transition, large mottles.

Section 2, $80 \mathrm{~cm}$ : cross-cut burrow.

Section 2, $97 \mathrm{~cm}$ : large fish bone.

Section 2, 107-108 cm: homogeneous.

Section 2, 112-117 cm: large thin burrow.

Section 2, 127-131 cm: homogeneous.

Section 2, 145-148 cm: silty, homogeneous.

Section $3,10 \mathrm{~cm}$ : trace diatomaceous ooze tufts.

Section $3,40 \mathrm{~cm}$ : minor tufts.

Section 3, 80-86 cm: transition burrows.

Section $3,84 \mathrm{~cm}$ : dark red concretion.

Section 3, 86-94 cm: wispy dark laminations, fold, unconformity.

Section 3, 109-112 cm: homogeneous.

Section 3, $117 \mathrm{~cm}$ : grayish orange diatomaceous ooze.

Section 3, 122-133 cm: thick pale lamination, $1 \mathrm{~mm}$.

CORE $7 \quad 28.50-33.25 \mathrm{~m}$

Section 1, 0-12 cm: disturbed.

Section 1, 12-30 cm: homogeneous.

Section 1, 30-34 cm: layered.

Section 1,34-38 cm: homogeneous.

Section 1, $40 \mathrm{~cm}$ : very fine lamination, burrows.

Section 1, 50-60 cm: mixed.

Section 1, 51-53 cm: homogeneous.

Section 1, 57-65 cm: large burrow.

Section 1, 75-78 cm: homogeneous, benthics.

Section 1,87 cm: sharp contact, stringers, benthics.

Section 1, 125-130 cm: homogeneous, burrows.

Section 1, $130 \mathrm{~cm}$ : angular discordance.

Section 1, 135-141 cm: homogeneous, burrow.

Section 2, $16 \mathrm{~cm}$ : several thin layers, lamination.

Section 2, 24-27 cm: lamination.

Section 2, 27-53 cm: gritty foraminifer bed.

Section 2, $42 \mathrm{~cm}$ : dark olive, convex structure.

Section 2, 58-59 cm: faint lamination.

Section 2, 88-92 cm: burrowed contact, lamination faint.

Section 2, 125-139 cm: bed with layering, foraminifer-rich base.

Section 3, 16-17 cm: layered, thin lamination, more than $20 \mathrm{~cm}$.

Section $3,30 \mathrm{~cm}$ : brownish rust, unconformity.

Section 3, 36-40 cm: ultra lamination.

Section 3, 40-50 cm: mixed, rich in diatom ooze.

Section 3, 69-70 cm: microbioturbated, speckled, bands.

Section 3, 81-82 cm: homogeneous.

Section $3,86-89 \mathrm{~cm}$ : transition.

Section 3, 93-98 cm: bone piece, gritty layers rich in foraminifers.

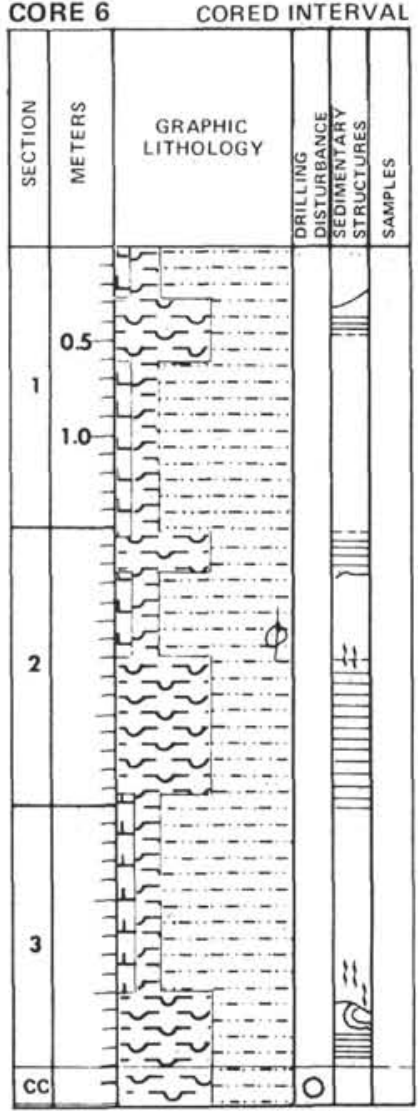

Figure 3. (Continued). 

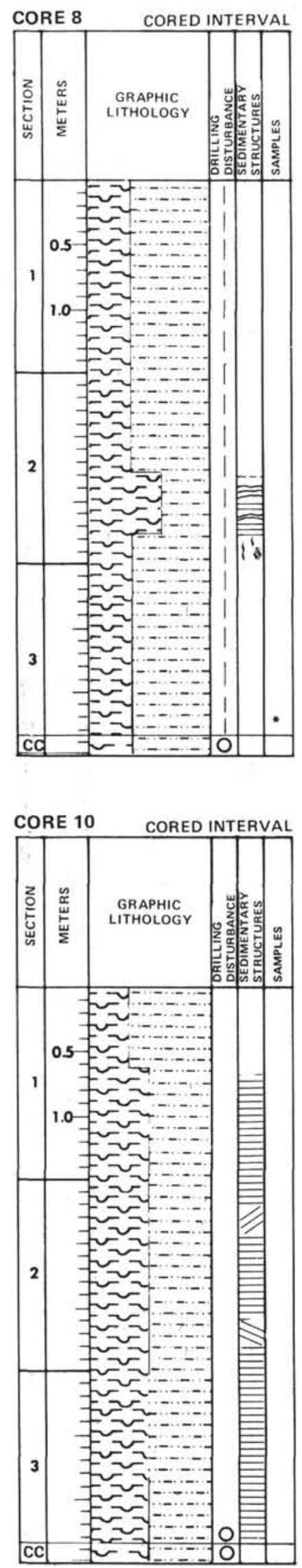

CORE $8 \quad 33.25-38.00 \mathrm{~m}$

Section 1, 0-19 cm: disturbed.

Section 1, 20-40 cm: tufts in homogeneous.

Section 1,146 cm: pumice.

Section 2, 0-20 cm: scattered organic bits, shell.

Section 2, $39 \mathrm{~cm}$ : brown fish vertebrae, gritty, glauconite.

Section 2, 40-80 cm: glauconite, patchy.

Section 3, 81-91 cm: transitional, some lamination, matted. Section $2,90 \mathrm{~cm}$ : disturbed lamination.

Section 2, 112-115 cm: homogeneous; lamination decays to $122 \mathrm{~cm}$.

Section 2, 122-150 cm: fine-grained mud.

Section 3, 38-40 cm: faint mottles.

Section 3, 80-90 cm: burrow mottled.

CORE $9 \quad 38.00-42.75 \mathrm{~m}$.

Section 1, 0-80 cm: uniform.

Section 1,84 cm: fish vertebrae.

Section 1, $109 \mathrm{~cm}$ : concretion.

Section 1, 120-124 cm: faint bands.

Section $2,0-5 \mathrm{~cm}$ : faint lamination.

Section 2, 40-60 cm: relict diatom tufts, pale.

Section 3, 0-20 cm: some mottles, large burrows.

Section $3,50 \mathrm{~cm}$ : bones.

Section 3, $84 \mathrm{~cm}$ : white specks.

CORE $10 \quad 42.75-47.50 \mathrm{~m}$

Section $1,0 \mathrm{~cm}$ : mud clasts.

Section 1, $30 \mathrm{~cm}$ : banded.

Section 1, $60 \mathrm{~cm}$ : discordant lamination.

Section $1,75 \mathrm{~cm}$ : thin shelled mollusk.

Section 1, $118 \mathrm{~cm}$ : thick, pale lamination.

Section 1, 17-20 cm: unconformity, pinch out.

Section 1, 20-30 cm: thick pale diatomaceous ooze, lamination.

Section 2, 30-34 cm: diffuse lamination.

Section $2,70 \mathrm{~cm}$ : very fine lamination, thick, pale.

Section 2, 100-110 cm: lamination well-developed, down increase in thick, pale.

Section 2, $110 \mathrm{~cm}$ : unconformity.

Section $3,10-20 \mathrm{~cm}$ : dark.

Section 3, 20-30 cm: two thick pale laminae.

Section $3,55-60 \mathrm{~cm}$ : lamination with thin gray top.

Section $3,92 \mathrm{~cm}$ : disturbed.

Section $3,98 \mathrm{~cm}$ : thick pale lamination.

Section $3,112 \mathrm{~cm}$ : tuffs.

Section 3, 115-122 cm: become faint.

\section{CORE $11 \quad 47.50-52.25 \mathrm{~m}$}

Section 1, 20-40 cm: thin-shelled mollusks.

Section 1,47 cm: large, $1 \mathrm{~mm}$, pearly fish scales.

Section 1, 60-116 cm: bands, some faint.

Section 1,88 cm: organic lamination.

Section 1, $116 \mathrm{~cm}$ : fish scales, mollusk, gas expansion cracks.

CORE $12 \quad 52.25-57.00 \mathrm{~m}$

Core-Catcher: encountered sand which was washed to $61.75 \mathrm{~m}$.
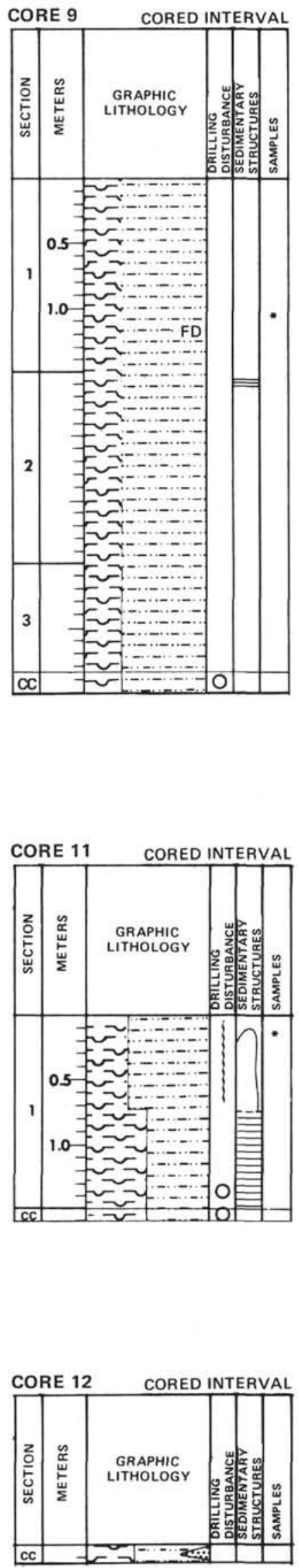

Figure 3. (Continued). 

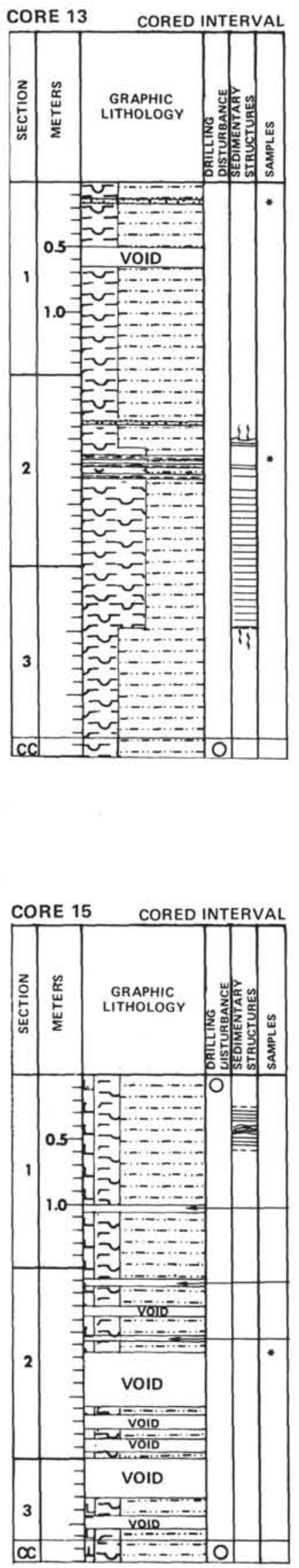

Figure 3. (Continued).
CORE $13 \quad 61.75-66.50 \mathrm{~m}$

Section 1, 0-20 cm: core disturbance.

Section 1,65 cm: scoria basalt pebble.

Section 1, 80-100 cm: gritty, white specks.

Section 1, $138 \mathrm{~cm}$ : burrow, finger-shape.

Section 2, 0-25 cm: burrow mottles.

Section 2, $29 \mathrm{~cm}$ : silty.

Section 2,34 cm: thin silty with fish debris, scales.

Section 2, $44 \mathrm{~cm}$ : sand patch.

Section 2,65-66 cm: thin sandy silt.

Section 2, 69-71 cm: homogeneous patch cuts laminated. Section $2,77 \mathrm{~cm}$ : thin gray clay.

Section 2, $78.5 \mathrm{~cm}$ : mud.

Section 2, 97.5-99 cm: homogeneous.

Section 2, 109-112 cm: homogeneous, white specks.

Section 2,119 and $123 \mathrm{~cm}$ : homogeneous.

Section 2, 134-139 cm: mats of pale diatomaceous ooze. Section 2, $146 \mathrm{~cm}$ : wood.

Section 3, 40-44 cm: transitional to homogeneous, abrupt. Section $3,111 \mathrm{~cm}$ : mottled.

\section{CORE $14 \quad 66.50-71.25 \mathrm{~m}$}

Section 1, 0-37 cm: layering cut by homogeneous.

Section 1,61 cm: large fish vertebrae.

Section 1, 70-80 cm: large cross burrows.

Section $1,110 \mathrm{~cm}$ : pumice fragment.

Section 1, $126 \mathrm{~cm}$ : debris.

Section 1, $128 \mathrm{~cm}$ : silicic wood?

Section 2, $50 \mathrm{~cm}$ : mottled.

Section 2, $130 \mathrm{~cm}$ : shell hash and chitin.

Section $3,10 \mathrm{~cm}$ : darker patches.

Section $3,38 \mathrm{~cm}$ : light in dark matrix.

Section $3,60 \mathrm{~cm}$ : shell hash.

Section $3,81 \mathrm{~cm}$ : mud clast with relict varve.

Section 3,94-117 cm: transitional to lamination.

\section{CORE $15 \quad 71.25-76.00 \mathrm{~m}$}

Section 1, 33-59.5 cm: lamination with disturbance, sharp contact.

Section $1,50 \mathrm{~cm}$ : unconformity.

Section 1, $55 \mathrm{~cm}$ : thin, rich in organic matter.

Section 1, 82-92 cm: scattered organic, white specks. Section 2, $147 \mathrm{~cm}$ : rounded cobble exotic.

\section{CORE $16 \quad 76.00-80.75 \mathrm{~m}$}

Section 1, 18-22 cm: top with scattered large homogeneous (burrow), pale laminae rich.

Section 1, 46-72 cm: darker zones dominant.

Section $1,80 \mathrm{~cm}$ : some diffuse lamination.

Section $1,100 \mathrm{~cm}$ : very fine, dark.

Section 1, 123-136 cm: thicker lamination.

Section 1, 139-140 cm: discordant bed.

Section 2: laminated to $16 \mathrm{~cm}$ then decline to $20 \mathrm{~cm}$, increase to $28 \mathrm{~cm}$, very fine at $24-25 \mathrm{~cm}$.

Section 2, 76 and $91 \mathrm{~cm}$ : fold.

Section 2, 97-98 cm: dark lamination, fish scales at 95 and $101 \mathrm{~cm}$.

Section $2,115 \mathrm{~cm}$ : thick tufts.

Section $3,1-2 \mathrm{~cm}$ : large fish scales.

Section $3,10 \mathrm{~cm}$ : fish scales.

Section 3,34 and $39 \mathrm{~cm}$ : fish scales, at $33.5 \mathrm{~cm}$ phosphate, white.

Section $3,51 \mathrm{~cm}$ : pale lamination, thick.

Section 3, $80.5 \mathrm{~cm}$ : pure white nannofossil lamination.

Section 3, 94-95 cm: very fine lamination.

Section 3, ? cm: dark in dark, very fine.
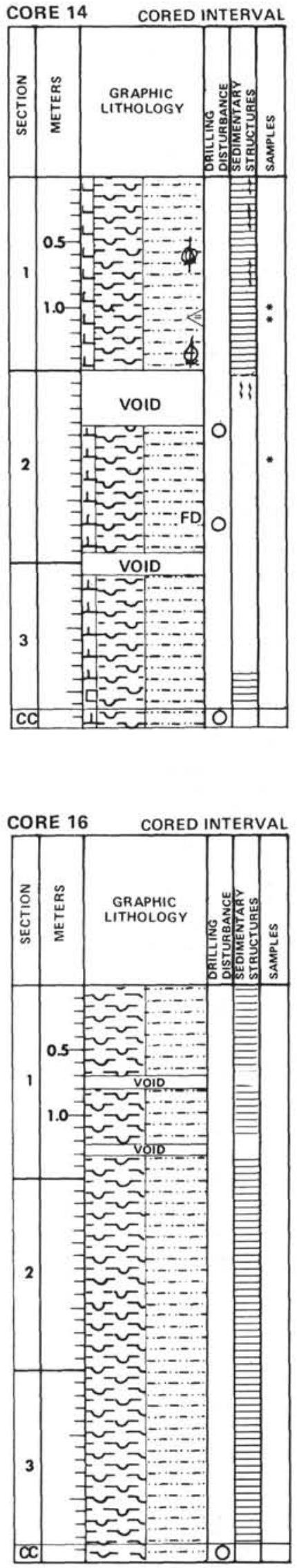

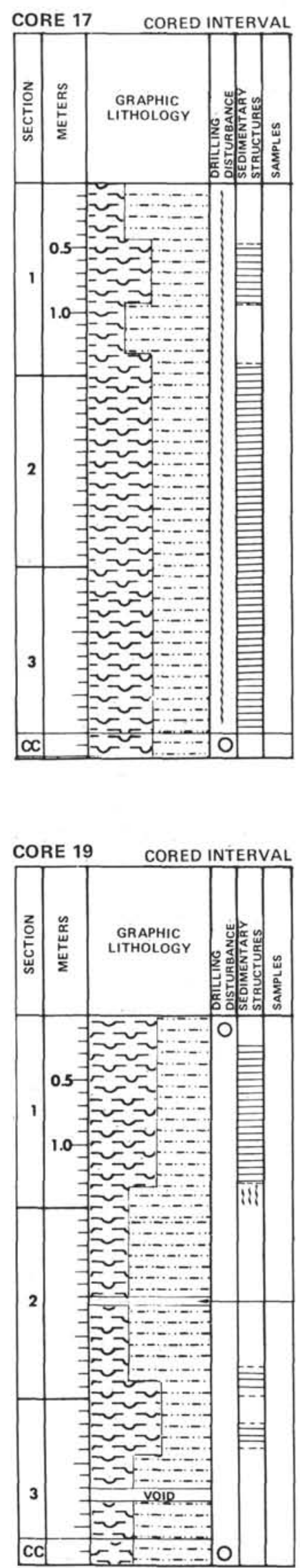

CORE $17 \quad 80.75-85.50 \mathrm{~m}$

Section 1: disturbed, inferred homogeneous foraminifer specks.

Section 1, $20 \mathrm{~cm}$ : faint bands; gritty.

Section 1, 40-70 cm: faint lamination interlayers.

Section 1, 105 and $112 \mathrm{~cm}$ : lamination.

Section 2, 0-125 cm: lamination disturbed by coring.

Section 3, 46-55 cm: homogeneous cross-splotch.

\section{CORE $18 \quad 85.50-90.25 \mathrm{~m}$}

Section 1, 0-18 cm: disturbed.

Section 1, 30-40 cm: lamination faint.

Section 1, 58-62 cm: homogeneous, faintly layered.

Section 1,88-92 cm: rich in pale lamination.

Section 1, 92-94 cm: homogeneous.

Section 1, 130-134 cm: thick multiple pale lamination. Section 1: several zones with common thicker pale diatomaceous ooze, laminae.

Section 2, $107 \mathrm{~cm}$ and below: lamination faint, crumbly.

CORE $1990.25-95.00 \mathrm{~m}$

Section 1, 0-23 cm: gas, faint.

Section $1,43-48 \mathrm{~cm}$ : very faint.

Section 1, 83-85 cm: thin detrital, thick pale diatomaceous ooze.

Section 1, 110-120 cm: transition, flasers, slumps.

Section 1, 120-154 cm: streaks, bands then homogeneous lighter color above.

Section $2,5 \mathrm{~cm}$ : mottles.

Section 2, $20 \mathrm{~cm}$ : banding.

Section 2, 40-60 cm: burrow mottles.

Section 2, 125-135 cm: transition evidence lamination, large brown chitin carapace.

Section 3, 0-14 cm: flakes, chips chitin.

Section 3, 18-22 cm: burrow cross.

Section 3, 25-40 cm: red brown to light brown chitin. Section 3, 40-60 cm: mottled.

Section $3,113 \mathrm{~cm}$ : large piece chitin.

CORE $20 \quad 95.00-99.75 \mathrm{~m}$

Section 1, 0-20 cm: disturbed light gray feldspar sand, coarse.

Section 1, $75 \mathrm{~cm}$ : pod-shaped burrow.

Section 1, 87-100 cm: mica-feldspar coarse gray sand,

Section 1, 114-126 cm: creep structure.

Section $1,134 \mathrm{~cm}$ : fold.

Section 2, 10-11 cm: thick, pale olive diatomaceous ooze.

Section 2, 24-26 cm: disrupted.

Section 2, 38-42 cm: sandy, graded bed, brown, full of fish debris.

Section 2, 97-99, 101, and $103 \mathrm{~cm}$ : burrow.

Section 2, 113-122 cm: convolute.

Section 1, $129 \mathrm{~cm}$ : burrow.

Section 1, 132-135 cm: very fine lamination, thick at 138 $\mathrm{cm}$.

Section 3, 9-11 cm: homogeneous. Below $10 \mathrm{~cm}$, increase in pale lamination thickness.
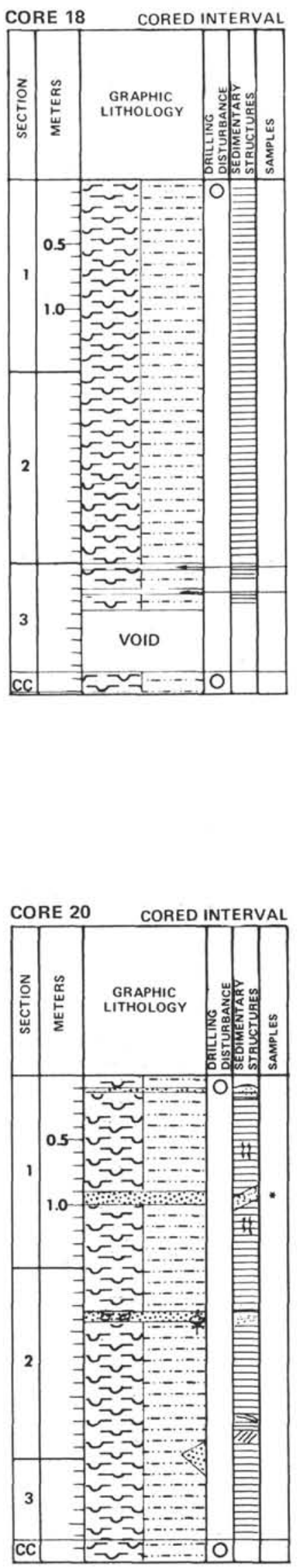

Figure 3. (Continued). 

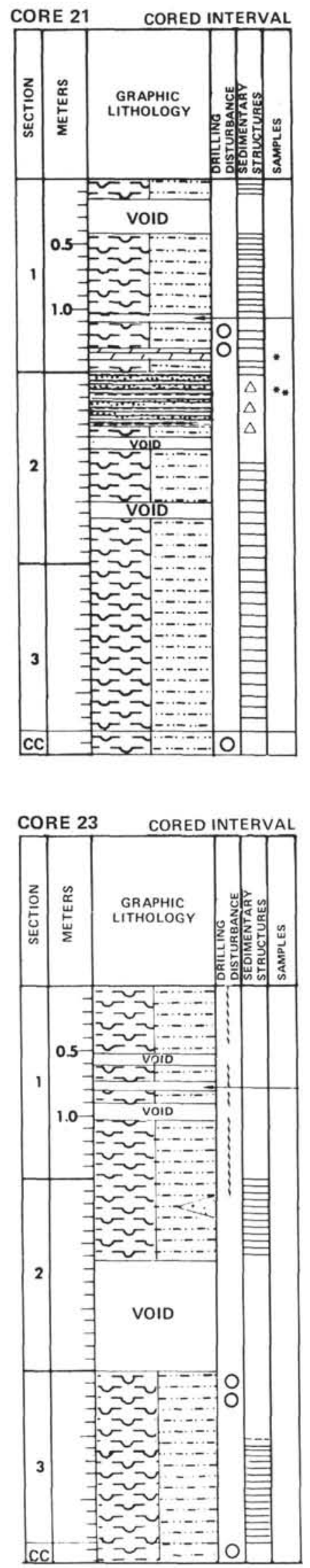

Figure 3. (Continued).
CORE $2199.75-104.50 \mathrm{~m}$

Section 1, $14.5 \mathrm{~cm}$ : shell.

Section 1,17.2 cm: gray bands.

Section 1, 41-42 cm: gray turbidite.

Section 1, 57-58 cm: gray graded bed.

Section 1,60 cm: change to broad banding.

Section 1, $75 \mathrm{~cm}$ : black lamination.

Section 1,81 cm: homogeneous to $86 \mathrm{~cm}$, lamination $89-91$ $\mathrm{cm}$, gray turbidite to lamination at $97.5 \mathrm{~cm}$.

Section 1, 126-141 cm: hard to soft dolostone.

Section 2, 0-60 cm: series of 54 thin gray beds, some graded sands, silts, interlayered, lamination, diatomaceous ooze, rhythmic.

Section $2,60 \mathrm{~cm}$ : burrows in sand.

Section $2,70-80 \mathrm{~cm}$ : increased burrowing mottles.

Section 2, 111-124 cm: gray bands.

Section 2, 119-120 cm: sand.

Section 2, 124-150 cm: homogeneous.

Section 2, $142 \mathrm{~cm}$ : hard, white concretion, thin single nannofossil laminae.

Section 3, 3-5 cm: mottled.

Section 3,28 cm: dark organic streaks.

Section $3,35 \mathrm{~cm}$ : chitin piece of crab, faint bands.

Section 3, 43-60 cm: lamination, some carbonaceous, 50 $\mathrm{cm}$ diffuse faint bands.

Section 3, 82-83 cm: laminae.

Section 3, 90-100 cm: lamination, ultra fine, dark.

Section 3, 101-110 $\mathrm{cm}$ : band 5 .

CORE 22 104.50-109.25 m

Section 1, 0-40 cm: disturbed.

Section 1, 40-50 cm: homogeneous with faint folds.

Section 1, 50-57 cm: lamination, faint to $78 \mathrm{~cm}$, thick pale at $82,85,88,94,96,99$, and $100 \mathrm{~cm}$, diminish below.

Section 1, $121 \mathrm{~cm}$ : transitional to homogeneous, mottled.

Section 1, $133 \mathrm{~cm}$ : dark band.

Section 1, 137-139 cm: faint lamination.

Section 2, $30 \mathrm{~cm}$ : large pieces, some fish debris, laminated.

Section 2, 57-58 cm: at $15 \mathrm{~cm}$ faint lamination, $58-68 \mathrm{~cm}$ graded silt.

Section $2,74 \mathrm{~cm}$ : thin white laminae.

Section 2, $91 \mathrm{~cm}$ : fish debris, 95-95 cm laminae.

Section 2, $102 \mathrm{~cm}$ : transitional to $104 \mathrm{~cm}$.

Section 2, 114-116 cm: homogeneous with chip of laminae.

Section 2, $135 \mathrm{~cm}$ : rich organic, black, woody.

Section 3, $14 \mathrm{~cm}$ : several thin pale diatomaceous ooze. Section 3, 0-23 cm: dark.

Section 3, 28-29 cm: homogeneous.

Section 3, 34-37 cm: homogeneous.

Section 3, 38-39 cm: unconformity, darker.

Section $3,57.7 \mathrm{~cm}$ : thin gray (ash?).

Section 3, 112-113 cm: minor homogeneous.

Section $3,118 \mathrm{~cm}$ : thick pale diatomaceous ooze.

Section $3,120 \mathrm{~cm}$ : thin crab shell.

\section{CORE 23 109.25-114.00 m}

Section 1, $10 \mathrm{~cm}$ : much gas separation, deformed.

Section 1, 28-30 cm: sand stringer.

Section 1, 110-115 cm: sand stringer.

Section $1,140 \mathrm{~cm}$ : very fine laminae.

Section 2, $6 \mathrm{~cm}$ : concretion, brown, soft, phosphate(?).

Section 2, 34-36 cm: sandy, brown.

Section 2, $60 \mathrm{~cm}$ : white diatomaceous ooze tufts.

Section 3, $10 \mathrm{~cm}$ : drill hash but chips show lamination.

Section 3, $91 \mathrm{~cm}$ : ultra fine laminae. Section $3,99 \mathrm{~cm}$ : gray band $2 \mathrm{~mm}, 100 \mathrm{~cm}$ slump.

Section 3,101 and $103 \mathrm{~cm}$ : thin white lamination.

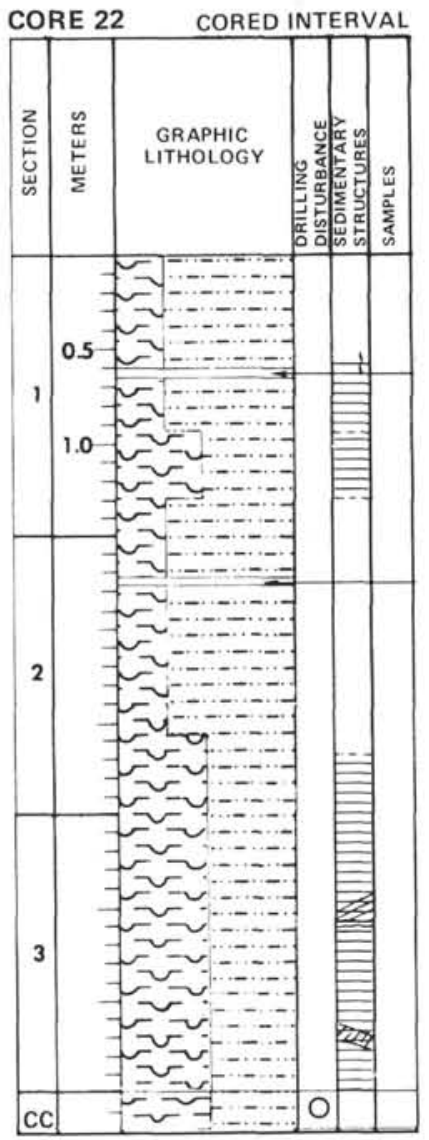

CORE 24: $109.25-114.0 \mathrm{~m}$

No recovery. 

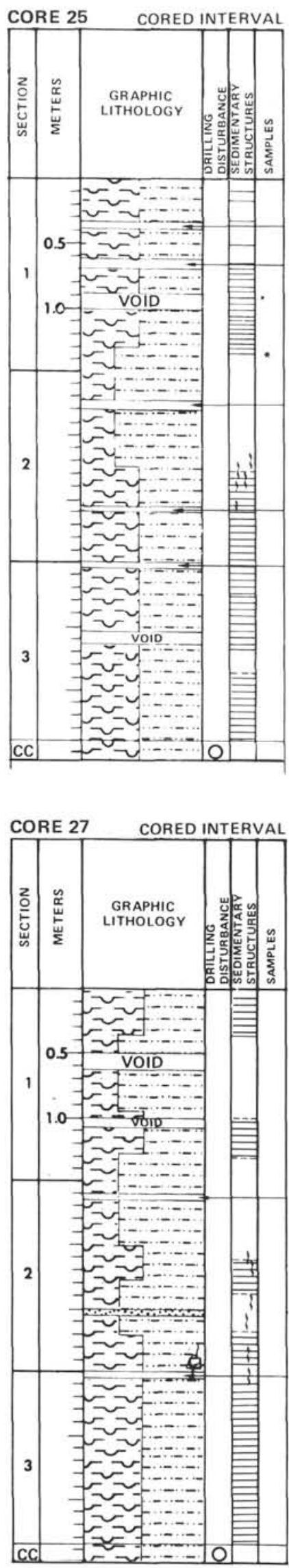

Figure 3. (Continued).
CORE $25 \quad 118.75-123.50 \mathrm{~m}$

Section $1,37 \mathrm{~cm}$ : faint lamination.

Section 1, $45 \mathrm{~cm}$ : dark, $48-51 \mathrm{~cm}$ fish debris.

Section 1, $53 \mathrm{~cm}$ : fish clay.

Section 1, 69-70 cm: 10 faint laminae, matted diatom.

Section 1, $75 \mathrm{~cm}$ : contact homogeneous to laminated, mixed.

Section 1, 103-113 cm: disturbed.

Section 1, $128 \mathrm{~cm}$ : contact to homogeneous, cream color to $131 \mathrm{~cm}$ faint mottles.

Section 2, 35-40 cm: brittle, concretionary, sand.

Section 2, $49 \mathrm{~cm}$ : chitinous debris.

Section 2, 59-63 $\mathrm{cm}$ : boundary to light mottle/crunchy flake.

Section 2, 60-70 cm: homogeneous interlayered with laminae.

Section 2, 72-74 cm: irregular.

Section 2, 80-90 cm: homogeneous and $94 \mathrm{~cm}$ to laminae.

Section 2, 110-114 cm: diffuse lamination, $113 \mathrm{~cm}$ brown carapace mixed with crossed burrow tracks.

Section 3, $15 \mathrm{~cm}$ : faint lamination.

Section 3, 34-37 cm: fish debris.

Section 3, 42-44 cm: homogeneous.

Section 3, 49-53 cm: homogeneous.

Section $3,85 \mathrm{~cm}$ : thin, rich in organic matter.

Section $3,99 \mathrm{~cm}$ : thin hard laminae.

Section $3,117 \mathrm{~cm}$ : microslump $4 \mathrm{~mm}$.

Section 3,122 cm: carapace chitin.

\section{CORE 26 123.50-128.25 m}

Section 1: mottled pale lamination common.

Section 2, $0 \mathrm{~cm}$ : mostly dark/dark.

Section 2, $30 \mathrm{~cm}$ : pale orange clay band, phosphatic nodule increase in diatom rich.

Section 2, $85 \mathrm{~cm}$ : concentration organic matter.

Section 2, 94-95 cm: organic (wood?).

Section 3: very fine laminae, then homogeneous.

\section{CORE 27 128.25-133.00 m}

Section $1,30 \mathrm{~cm}$ : sharp contact, mud, faint layers.

Section 1, 32-50 cm: mud, dark brown.

Section 1, 84-90 cm: faint layering.

Section 1, $115 \mathrm{~cm}$ : mottles with trace laminae, evidence was laminated.

Section 2, $15 \mathrm{~cm}$ : dark olive brown.

Section 2, $51 \mathrm{~cm}$ : clast with laminae, large burrows.

Section 2, 60-74 cm: laminated, many diatomaceous ooze laminations, at $75 \mathrm{~cm}$ sharp contact to homogeneous.

Section 2, 110-120 $\mathrm{cm}$ : transition with large burrows.

Section 2, 140-147 cm: oblique finger burrow, wood.

Section 3, $24 \mathrm{~cm}$ : burrow.

Section 3, 32-39 cm: loss of pale olive laminae.

Section $3,39-42 \mathrm{~cm}$ : rich pale olive laminae, $46-50 \mathrm{~cm}$.

Section $3,50-67 \mathrm{~cm}$ : pale rare.

Section $3,117-117.5 \mathrm{~cm}$ : bioturbated, lamination uniform.

\section{CORE $28 \quad 133.00-137.75 \mathrm{~m}$}

Section 1: lamination uniform.

Section 1, 107-109 cm: white, gray pumice, rhyolite ash.

Section 2: overprint of $20 \mathrm{~cm}$ lighter to darker cycle, very little variation.

Section 3, 2-3 cm: mud clast.

Section 3, $31 \mathrm{~cm}: 8-10$ thicker pale olive.

Section 3, 44-45 $\mathrm{cm}$ : black pigment, $47 \mathrm{~cm}$ black.

Section 3, 59-60 cm: discontinuous.

Section 3,65-66 cm: enterolithic fold.

Section 3,95 cm: diatom-rich; fainter to $105 \mathrm{~cm}$.

Section $3,108-114 \mathrm{~cm}$ : homogeneous.
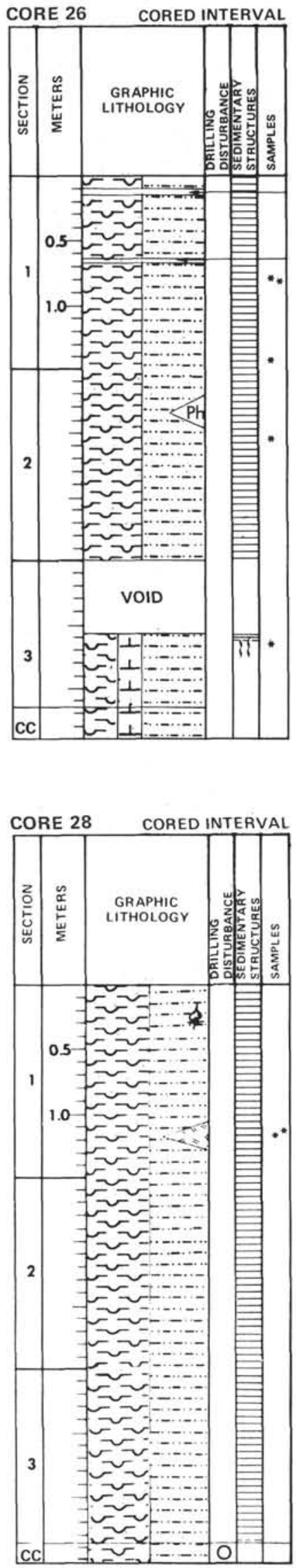


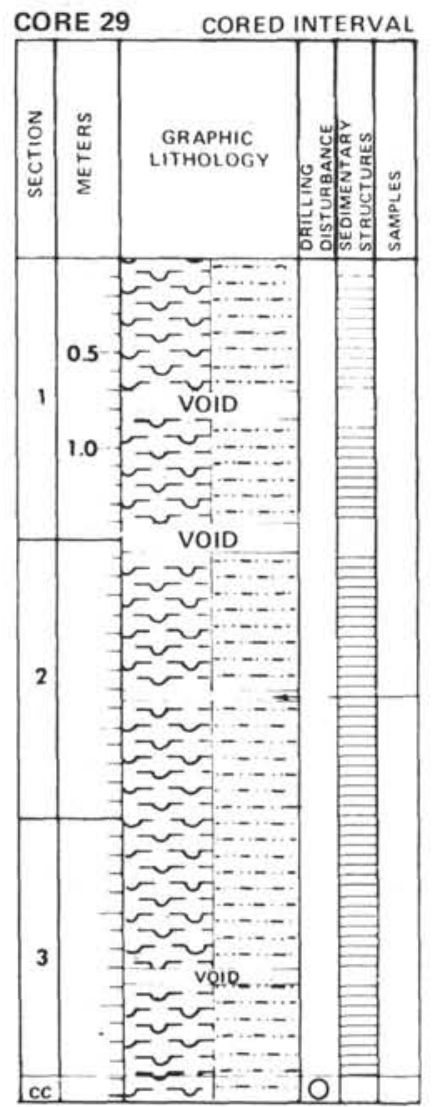

CORE $29 \quad 137.75-142.50 \mathrm{~m}$

Section 1, 0-16 cm: homogeneous, banded.

Section 1, 23-26 cm: homogeneous.

Section 1: dark laminae common, gradual increase down in pale olive, benthics and fish debris rare.

Section 2, $70 \mathrm{~cm}$ : transition to more detrital, laminae finer.

Section 2, 120-135 cm: pulses pale lamination.

Section $3,0-25 \mathrm{~cm}$ : darker, extreme fine.

Section 3, $21 \mathrm{~cm}$ : discordant.

Section 3, 30-33 cm: dark.

Section 3, 56-57 cm: microbioturbated.

Section $3,110 \mathrm{~cm}$ : terrigenous thin/pale olive thick.

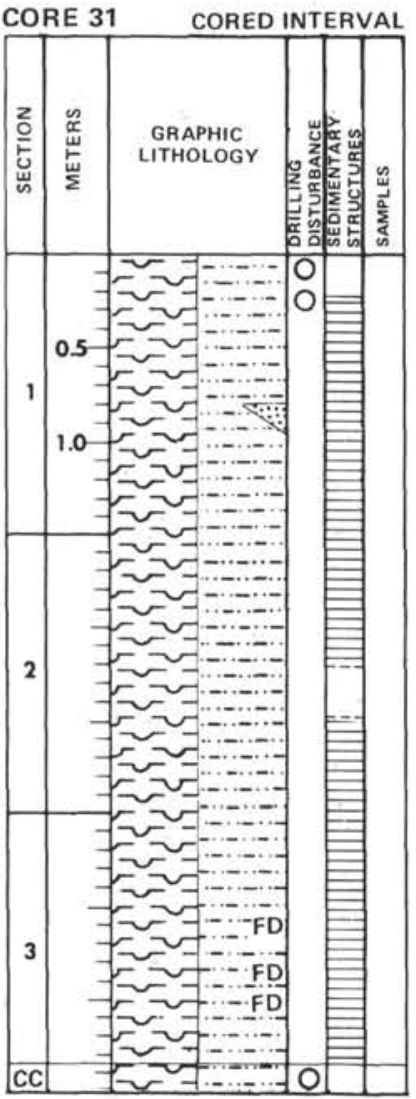
than light. foraminifers.
CORE 30 COREDINTERVAL

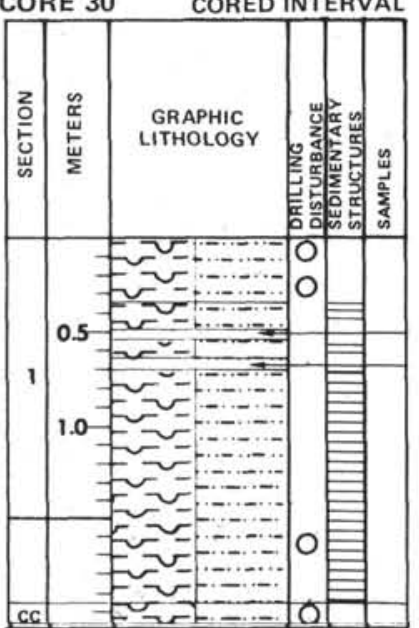

CORE $30 \quad 142.50-147.25 \mathrm{~m}$

Section 1, $45 \mathrm{~cm}$ : dark facies but terrigenous dark, is finer

Section 1, 128-130 cm: homogeneous.

Section 1, $137 \mathrm{~cm}$ : transitional to darker.

Section $2,10 \mathrm{~cm}$ : very fine laminae.

Section 2, $22 \mathrm{~cm}$ : flasers.

CORE $31 \quad 147.25-152.00 \mathrm{~m}$

Section 1, 50-51 cm: burrow homogeneous.

Section $1,84 \mathrm{~cm}$ : sand.

Section 1, 86-101 cm: sand along a crack.

Section 2, $5 \mathrm{~cm}$ : lamination mostly darker.

Section 2, $12 \mathrm{~cm}$ : bit of organic matter.

Section 2, $58 \mathrm{~cm}$ : wood, at $62-64 \mathrm{~cm}$ homogeneous with

Section 2, 70-84 cm: clay top, 84-90 cm faint layers. Section 2,90.5-95 cm: pinch out.

Section 2, $103 \mathrm{~cm}$ : diatom rich.

Section $3,6.5 \mathrm{~cm}$ : fish bone; lamination thin $(0.1-0.2 \mathrm{~mm})$. Section 3, $65 \mathrm{~cm}$ : fold.

Section 3, $84 \mathrm{~cm}$ : fish bones.

Figure 3. (Continued).

banding and differentiation of components. These are also shown in radiographs (see Soutar et al., this volume, Pt. 2). Some zones could be variously interpreted as layered or burrowed.

Typically, the lower contact of a thicker homogeneous bed is gradational, whereas the upper contact is commonly abrupt (see Fig. 7). This would be expected if, during times of increasing surface productivity, critically low bottom-water oxygen values suddenly exterminated a bottom fauna.

There is no evidence, such as basal sands or subtle grading, to conclude that homogeneous sections are redeposited sediments. There is evidence, however, of reworking of some foraminifers (Shackleton, this volume, Pt. 2). We interpret these zones to reflect times of a less pronounced oxygen-minimum zone in which the bottom conditions could support both an epifauna and a limited infauna. Along the lower contact, some zones could be variously interpreted as either layered or burrowed and disturbed. Indistinctly laminated patches are crosscut discordantly with homogeneous, brownish, diatom muds. These muds do not appear to be injections from the coring process, because the transition to laminations is abrupt, without deformation or signs of fracture. Instead, we interpret these transition zones to be burrowing pathways of large animals such as crabs or mollusks, which forage deeply and quickly through the anoxic sediments. Typically they occur near the lower contact of a homogeneous zone, which would be expected if an epifauna reappeared after more oxygenated conditions were restored on the sea floor. Such zones indicate that portions of the homogeneous sections may originally have been laminated and were later bioturbated, whereas other portions were continuously churned by infauna during sedimentation. A characteristic of the laminated zones which may have been later burrowed by larger 


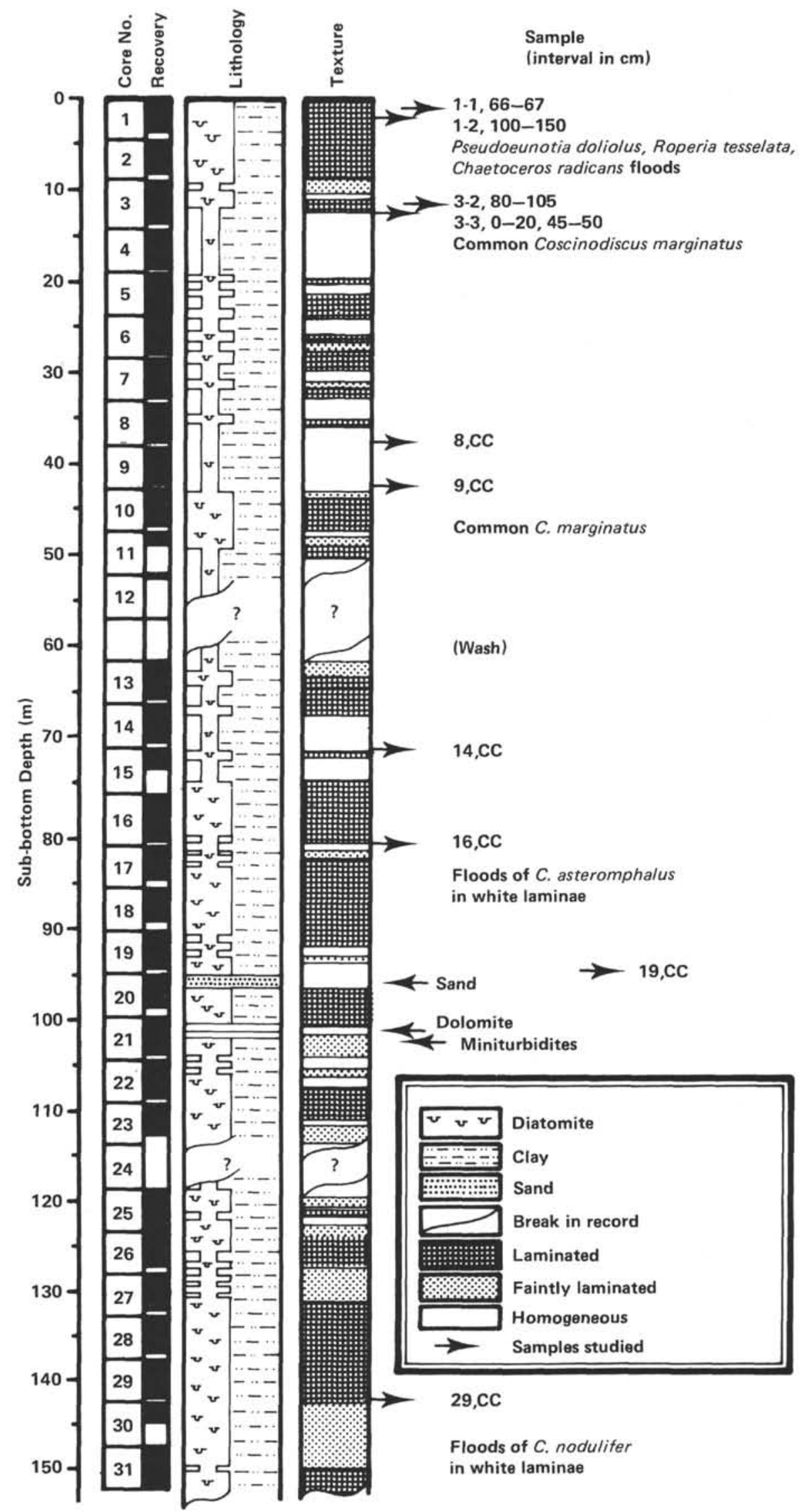

Figure 4. Site log of HPC Site 480. (From Schrader et al., 1980.) 

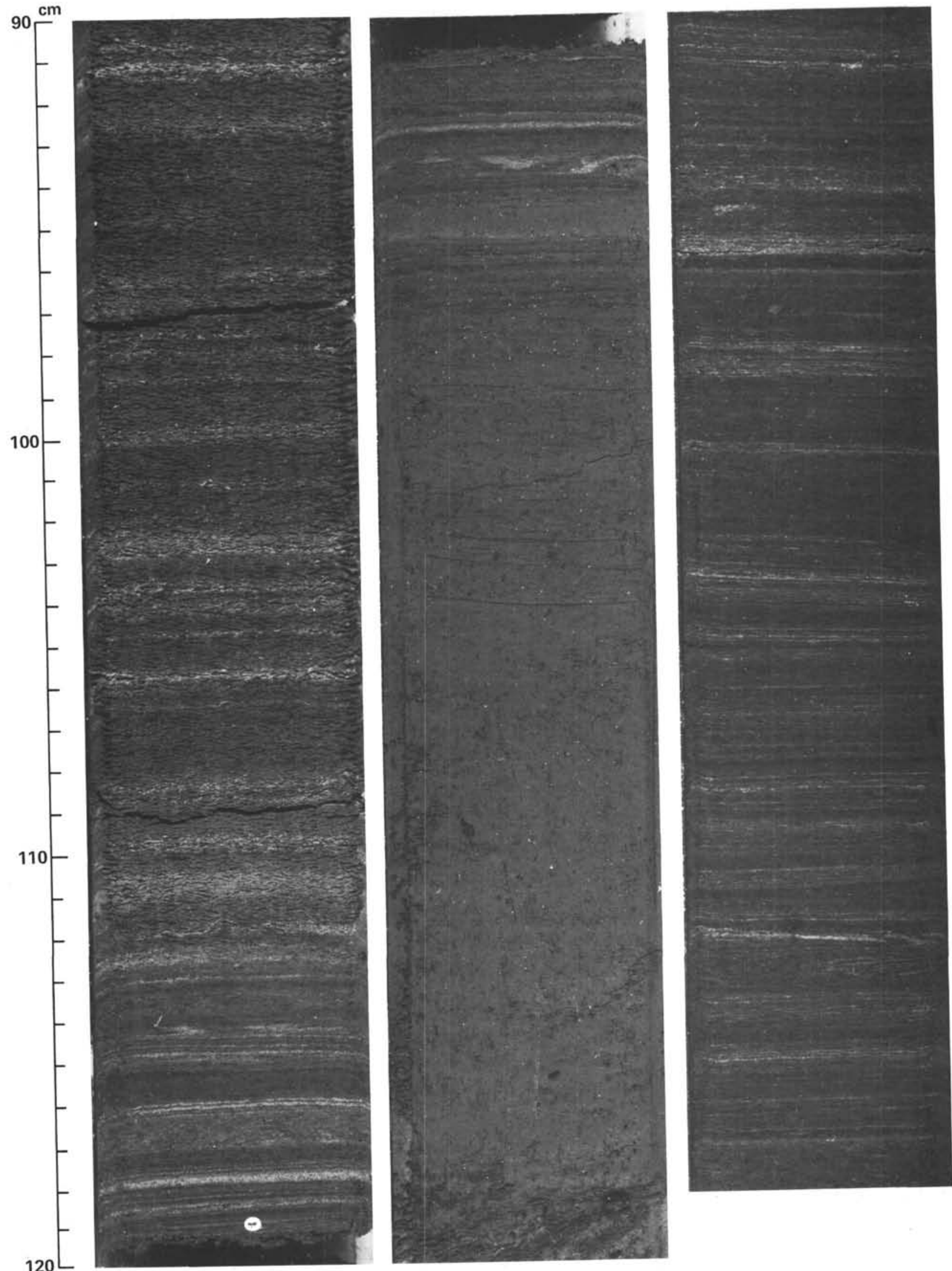

Figure 5. Comparison of core photographs from laminated and homogeneous sections of Sample 480-19-1, 90-120 cm. Some laminations show evidence of partial destruction. 
Table 1. X-ray diffraction survey of bulk sediment composition, Hole 480 .

\begin{tabular}{|c|c|c|c|c|c|c|c|}
\hline \multirow{2}{*}{$\begin{array}{c}\text { Sample } \\
\text { (interval in } \mathrm{cm} \text { ) }\end{array}$} & \multicolumn{4}{|c|}{ Height of Main Peak Reflex (mm) } & \multirow{2}{*}{$\begin{array}{l}\text { Minor } \\
\text { Clay }\end{array}$} & \multirow[b]{2}{*}{ Other } & \multirow[b]{2}{*}{ Amorph. } \\
\hline & Quartz & Calcite & Plagioclase & K-Feldspar & & & \\
\hline \multicolumn{8}{|l|}{ Hole 480} \\
\hline $\begin{array}{l}4, \mathrm{CC} \\
\text { (homogeneous) }\end{array}$ & 210 & 80 & 50 & 15 & $\begin{array}{l}\text { Broad, tr. ill, } \\
\text { chl, sm }\end{array}$ & Pyrite 6 & + \\
\hline $8, C C$ & 215 & - & 75 & 5 & $\begin{array}{l}\text { Broad, tr. sm, } \\
\text { ill }\end{array}$ & & ++ \\
\hline $\begin{array}{l}10, \mathrm{CC} \\
12, \mathrm{CC}\end{array}$ & 101 & 50 & 15 & 8 & III, chl, sm & Dolomite $(2.90 \AA) 21$, pyrite 10 & ++ \\
\hline (brown, sandy) & 220 & - & 112 & 122 & III $12, \mathrm{chl}$ & Amphibole, zeolite (clinop.) tr & - \\
\hline $14, \mathrm{CC}$ & 220 & 105 & 42 & 35 & Broad chl, sm & Dolomite 25 & ++ \\
\hline $18, \mathrm{CC}$ & 82 & - & 15 & 18 & III 12, chl, sm & Pyrite 14 & ++++ \\
\hline $20, \mathrm{CC}$ & 120 & - & 25 & 13 & $\begin{array}{l}\text { Ill 20, chl broad, } \\
\text { sm }\end{array}$ & $\mathrm{Mg}-\mathrm{CC}$ tr $(2.93,2.99)$, & ++ \\
\hline $22, \mathrm{CC}$ & 133 & - & 35 & 25 & Broad chl, ill, sm & & +++ \\
\hline $26, \mathrm{CC}$ & 220 & - & 40 & 34 & $\operatorname{Sm~12,~ill~12,~chl~}$ & $\mathrm{Mg}-\mathrm{CC}$ tr $(2.95)$ pyrite to & ++ \\
\hline $30, \mathrm{CC}$ & 183 & - & 29 & 20 & Sm 35, broad & $\begin{array}{l}\mathrm{Mg}-\mathrm{CC}(2.98 \AA) \text {, tr } 12, \text { tr } \\
\text { glauconite(?) }\end{array}$ & \\
\hline \multicolumn{8}{|l|}{ Hole 481} \\
\hline $8-3,1-3$ & 52 & - & - & - & Chl 65 , ill 20 & & \\
\hline $8-3,9-10$ & $230+$ & - & - & - & Chl 25 , ill 2 & & \\
\hline
\end{tabular}

Note: ill $=$ illite, $\mathrm{chl}=$ chlorite, $\mathrm{sm}=$ smectite, $\mathrm{tr}=$ trace.

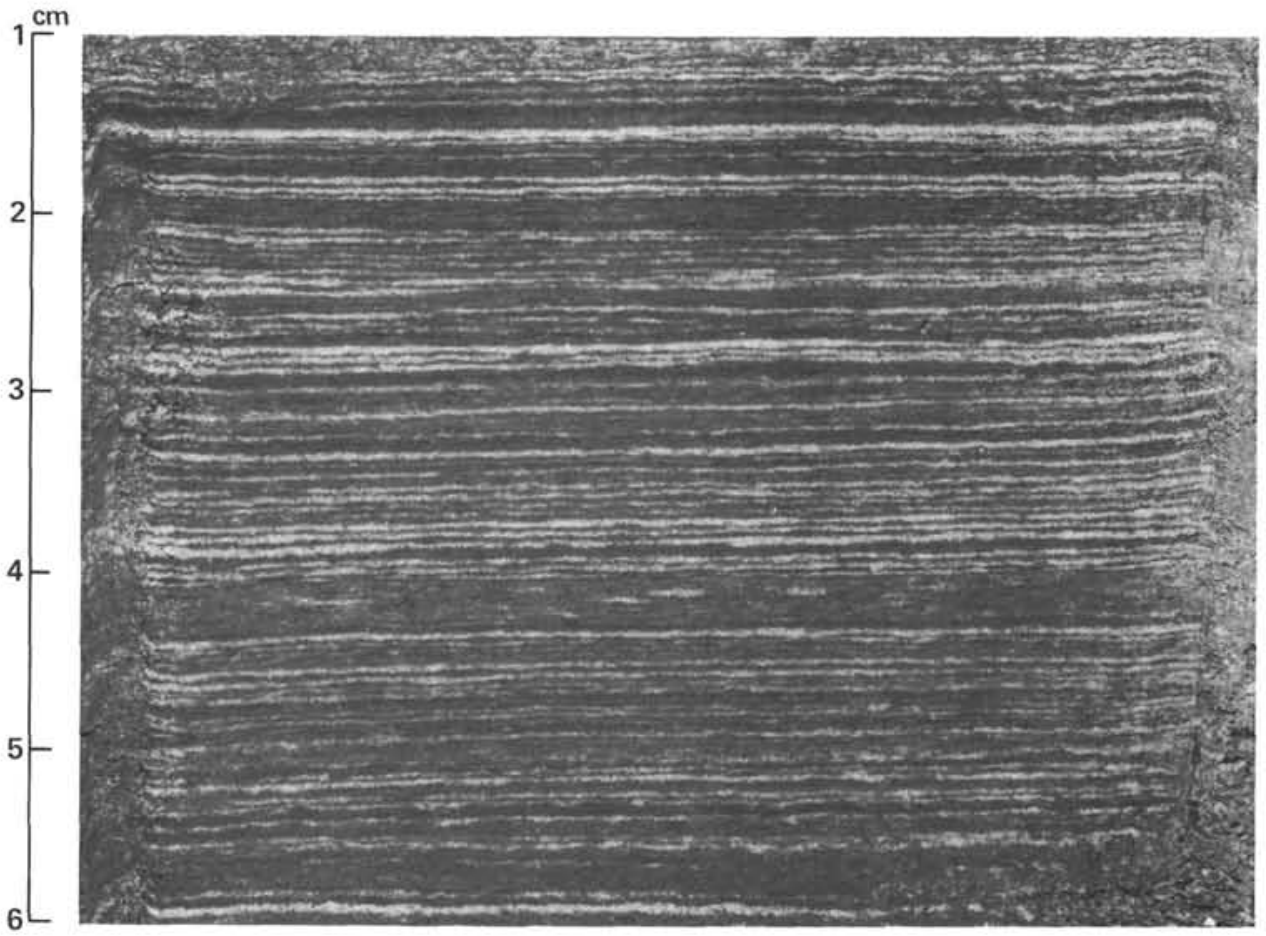

Figure 6. Detail of laminations from Sample 480-29-3, 145-150 cm, near base of a core, which has oceanic species in pale layers (Schrader, this volume, Pt. 2).

animals is that they contain significantly fewer benthic foraminifers. Some evidence is provided by scattered pieces of crablike carapace hash which occur exclusively in homogeneous zones. A boundary condition is documented by $\mathrm{cm}$-thick, nonlaminated layers which show relict evidence that the seasonal signal has been only barely disrupted.

\section{Events}

Interruptions within rhythmic patterns or the alternation of homogeneous and laminated zones are not common. Some thin layers show enterolithic folds and discordances, affecting only a dozen or so laminae (e.g., Fig. 8). Some have sharp chevron kink-folds which are 


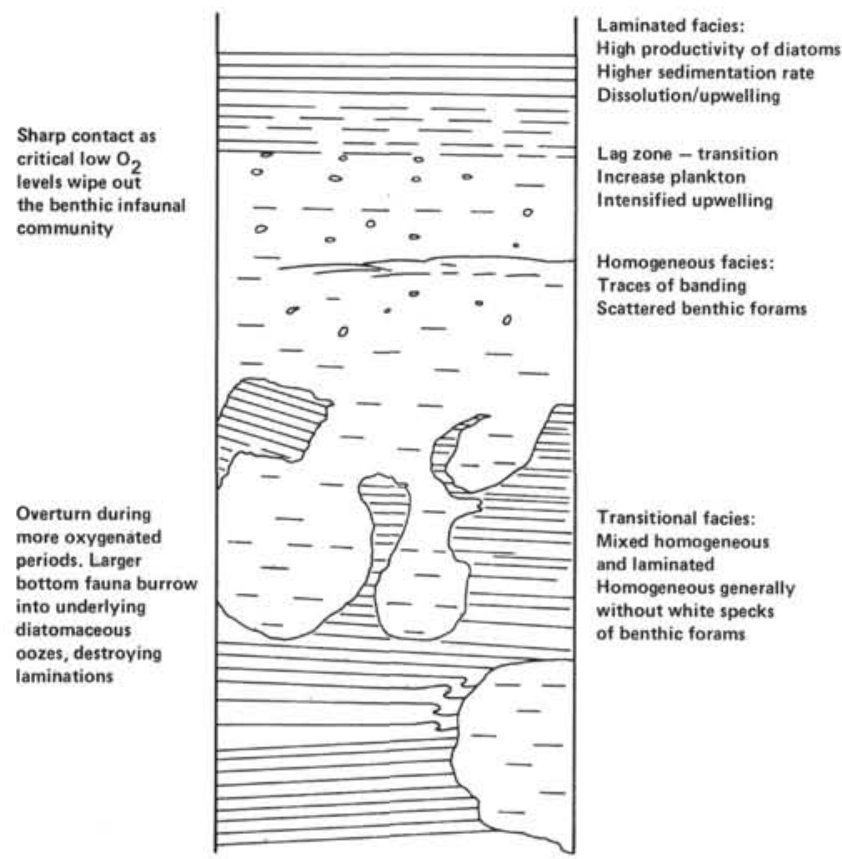

Figure 7. Generalized model of contact relationships for laminated and nonlaminated zones.

interpreted as possible seismites, caused by tectonic jolts transmitted in the soft surface layer. Degassing fractures and partings are common in lower cores.

A few undisturbed, medium gray, well-sorted, sand to silt intercalations occur in Core 20 (97 m; see Fig. 8) and as blebs in the poorly recovered core catcher of Core $13(67 \mathrm{~m})$. Their composition is typical of the Sonora Province (Aguayo, this volume, Pt. 2), immature with high percentages of plagioclase. Grains are angular to subangular (see Plate 1), dominantly quartz and feldspar with accessory epidote, hornblende, pyroxene, biotite, and volcanic fragments. The composition differs from the Yaqui River load, and appears to be derived instead from nearby coastal areas (Aguayo, this volume, Pt. 2). Upper and lower contacts are sharp, without grading or obvious internal organization, and as such might be products of sand falls or winnowing. One $3-\mathrm{cm}$ graded layer which might be a turbidite occurs as an olive brown sandy silt with coarse basal organic and shell debris, intercalated into a laminated zone (Section 480-20-2, Fig. 9). In Core $21(101 \mathrm{~m})$ a series of 45 cyclic, thin $(1-3 \mathrm{~cm})$, imperfectly graded, gray sand to silt beds occur just below a $10-\mathrm{cm}$ thick dolomitic mudstone (Fig. 10), but the origin of this sequence is puzzling. The basal sands are well sorted, with faint current laminations which are abruptly overlain by silty-clay tops. Individual beds are commonly separated by a few rhythmic diatomaceous couplets. The composition of the sandy parts is similar to other massive sand layers.

The 10-cm dolomite layer (Sample 480-21-1, 130-140 $\mathrm{cm}$ ) is a hard but friable yellowish, gray, fine-grained, structureless bed showing a few burrow traces. A similar bed is also suspected at Core $24(115 \mathrm{~m})$, where drillers' records indicate another hard layer. As shown by Kelts and McKenzie (this volume, Pt. 2), the dolomite layers are a diagenetic feature. Preliminary studies (R. Byrne, pers. comm.) suggest that the dolomite samples contain pollen from warmer climates than surrounding muds. If there is an association of sites of preferred dolomite nucleation and interglacial climates, then the concentration of sandy layers within Cores 20 and 21 could indicate times of rapid deglaciation and correspondingly more humid conditions as sea levels rose, flooding sandy coastal zones. We correlated these intervals to sandy and dolomitic zones of nearby Hole 479 .

Figure 3 also records the stratigraphic location of other features that indicate significant oceanographic fluctuations. Fish debris is conspicuous at several levels; Cores $7,8,9,16,22,26$, and 31 are particularly rich in bone material. Large vertebrae and bone fragments occur commonly in homogeneous facies. Fish scales also form microlaminae within many of the varve couplets (Schrader, this volume, Pt. 2). In Cores 7, 8, and 9, fish debris occurs together with significant glauconite pellet concentrations (Sample 480-8-2, 40-80 cm). Soft, yellowish orange phosphatic pellets and a thin phosphatic layer were found in Samples 480-9-1, $108 \mathrm{~cm}$, and 480-26-3, 60-140 cm. The enrichment in fish debris and phosphate perhaps coincide with apparently low sea level stands, when the site was closer to the upper boundary of the oxygen-minimum zone.

A thin, light gray, silty, rhyolitic ash present as pumice fragments in a matrix occurs at Sample 480-28-1, 107 $109 \mathrm{~cm}$, but has not been dated. Shards are probably wind-transported and are partially altered. Rhyolitic ash in Hole 479 at a similar depth $(134 \mathrm{~m})$ might be contiguous, but that is not yet certain (Plate 1). Fragments of altered basaltic ash with manganese-coated grains were found in Sample 480-14-1, $110 \mathrm{~cm}$. These sporadic layers may have been redeposited from coastal areas where both young rhyolites and basalts outcrop.

Although the cores are generally low in carbonate, some curious thin laminae are encountered, for example in Cores 480-21 and 28; these consist exclusively of the robust nannofossil species, Coccolithus pelagicus (Aubry, this volume, Pt. 2). Their distribution and their origin (are they the result of blooms or dissolution?) are questions still under study.

\section{DISCUSSION}

The general sedimentation patterns of Site 480 have remained fairly uniform throughout the cored interval. An interpretation of the historical development of the sediment sequence in terms of oceanographic changes and glacial cycles is not possible without a precise time framework which we have not yet unequivocally established, in spite of the studies presented in this section. Several lines of evidence point to maximum ages of from 200,000 to 400,000 y. (Schrader, this volume, Pt. 2). Varve counts (Soutar et al., this volume, Pt. 2) at least seem to confirm that most couplets are annual. The section documents seasonal hemipelagic sedimentation on an outer slope which is situated below a fertile, productive water mass. Scattered glauconite, phosphate, and some fish debris concentrations provide signs of times with relatively lower sedimentation rates, possible 

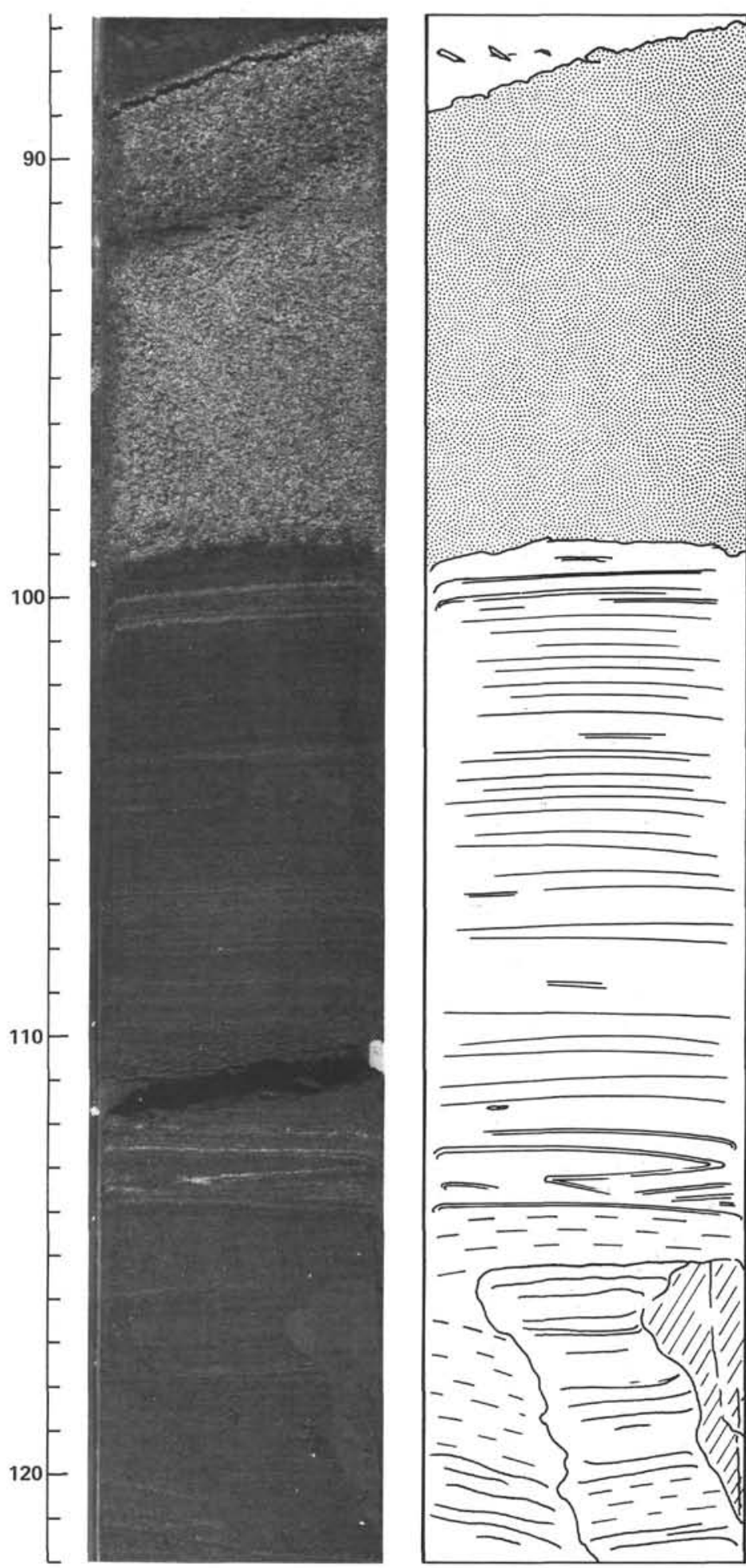

Darker olive brown

laminated muddy

diatom ooze

Massive, uniform,

well-sorted

gray sand with

sharp contacts

Several thicker,

pale olive,

diatom-rich

laminae

Zone of finely laminated diatom mud with only rare thicker pale-olive diatom-rich laminae

Enterolithic fold with pale olive laminae

Thin homogeneous zone with forams

Partly burrowed

laminated zone with large burrow patches

Faint laminations

in tilted host

Figure 8. Sample 480-20-1, 87-122 cm, with interpreted description.

in conjunction with minor winnowing. These alternate with times of higher rates marked by particularly diatom-rich oozes. The occurrence of some reworked foraminifers, for example in Core 480-8 (Shackleton, this volume, Pt. 2) at levels coinciding with glauconite pellets and glacial conditions, might provide evidence for winnowing during times of lowered sea level. A mechanism for this is unclear, because a sea level change of only 100 meters or so would not significantly affect the depth setting of Site 480 . In a similar vein, sand layers and minor discordances may indicate a short hiatus of unknown duration in Cores 480-20 and 21. 


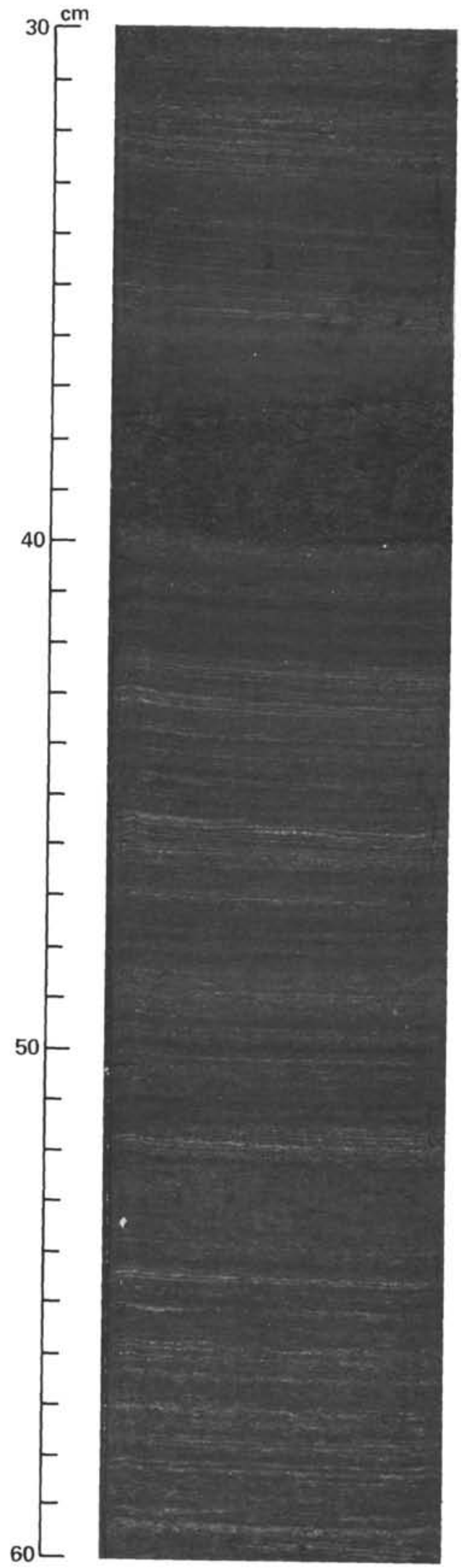

Figure 9. Laminated section (Sample 480-20-2, 30-60 cm) with a rare small, olive-brown graded sand to silt bed, also containing coarse organic debris.

\section{Varve Formation}

The Guaymas Basin at present is noted for high organic productivity (Revelle, 1950; Zeitschel, 1969), particularly of diatoms associated with coastal upwelling triggered by northwesterly winds during the dry season (January through May). A pale olive lamina with an excellently preserved upwelling diatom assemblage is produced during that season. Upwelling along the Guaymas Slope ceases during the rainy season (July through September) when winds come from the southeast (Roden, 1964) and terrigenous material can be transported into the area from the Yaqui or other Sonoran rivers to form a dark lamina with a moderately well preserved oceanic diatom assemblage. This simple couplet compositional pattern, although prevalent in the first few cores, is contradicted in other parts of the section at Site 480 . In Cores 480-16 (78 m) and 480-29 (142 m), for example, some light laminae contain monospecific diatom assemblages of Coscinodiscus asteromphalus and $C$. nodulifer, which are oceanic forms (Schrader, this volume, Pt. 2; Schrader et al., 1980). Darker laminae are dominated by an upwelling-type flora. Such shifts suggest complex interactions of climate and ocean currents but they will eventually allow us to resolve major patterns in the Gulf region.

A further implication of the wind-current model of varve formation is that regionally it should be diachronous and at some point the input signals should cancel. This has not yet been documented. Calvert (1966) was not able to correlate individual layers although he found laminated oozes rimming the Guaymas Basin in a girdle from 300-1200 meters depth. The following criteria were applied to derive a model of variable terrigenous input (Fig. 1): (1) Light and dark laminae contain essentially the same assemblages. (2) Light laminae are composed of $15-75 \%$ opal, dark of $8-50 \%$ opal. (3) Muds are laminated near river mouths in spite of low diatom content. (4) Dark laminae vary in thickness and in some cases show size grading. (5) Diatoms are abundant in water samples throughout the year.

Calvert's 1966 model implied that darker layers should be thicker; this has not been generally observed for Hole 480. Radiography (Soutar et al., this volume, Pt. 2) documents the occurrence of complex sublaminae. Thick, pale olive laminae may actually represent several years of drought in some cases-one of the pitfalls inherent in varve counts. At present, we conclude that the modus of varve formation may vary regionally. Indeed, the key to understanding both laminated and nonlaminated facies as well as the first-order formation of varves may be rainfall patterns. Soutar and Crill (1977) found that the thickness of varves in the Santa Barbara Basin correlates with rainfall in the same manner as tree rings do.

Laminated sections also have second-order variations that require further examination. Couplets are grouped into banded rhythms of darker and lighter predominant hues on a decimeter scale. Sample counts suggest 20-100 y. fluctuations. These may be in concert with modest variations in intensity of upwelling coupled with drier 


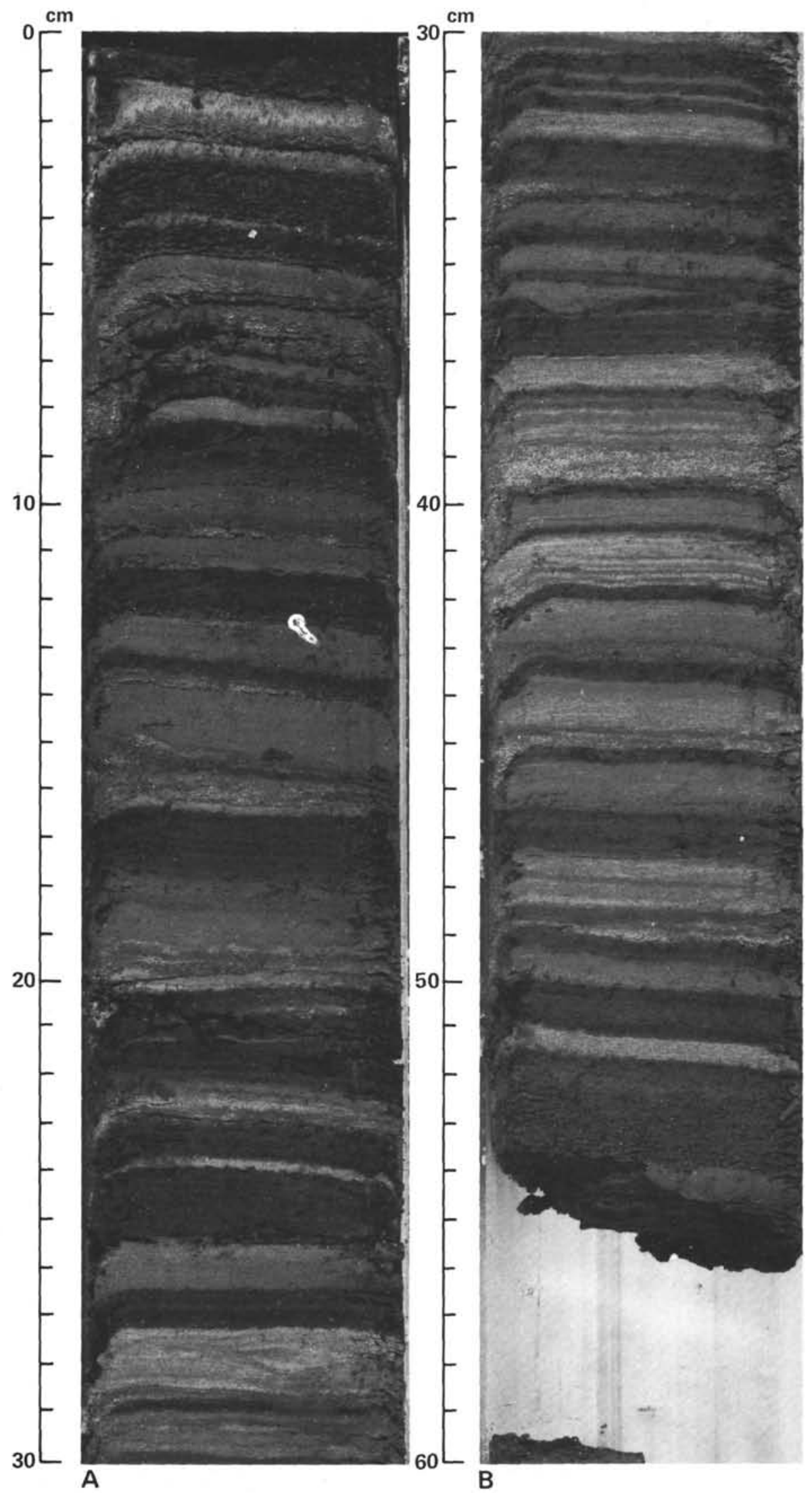

Figure 10. Clastic rhythms sequence, Samples 480-21-2, 0-30 cm (A) and 30-60 cm (B). Thin light gray silts to sand, partly graded with intercalations of host (dark) diatomaceous muds. Basal sand fractions partly winnowed. 
and wetter spells landward, as is suggested in the pollen spectrum (Heusser, this volume, Pt. 2).

\section{Laminated and Nonlaminated}

For the uppermost 15 cores of Hole 480 , subsequent studies confirm that the nonlaminated zones are generally correlated with glacial stages on land. They contain both more cold-water diatom species (Schrader, this volume, Pt. 2) and pollen from cooler periods (Heusser, this volume, Pt. 2; Byrne, this volume, Pt. 2). It is not yet clear how climate and upwelling are interrelated. The question arises whether nonlaminated zones represent times with significantly reduced upwelling or whether Hole 480 is merely recording a minor vertical displacement in the locus of the oxygen-minimum water mass. A hydraulic piston coring transect is needed to clarify this picture, but some observations suggest that the zones record major changes in circulation patterns.

The palynological spectrum, for example, changes across the Pleistocene/Holocene boundary, which is also a change from homogeneous to laminated facies. Pinus, an indicator of wetter landward climate, is more common below Core 480-3, in nonlaminated facies, but paradoxically dinoflagellates, commonly associated with upwelling, are also abundant there. The landward climate was drier for the laminated Holocene deposits (Heusser, this volume, Pt. 2). Simoneit (this volume, Pt. 2) also found that although the lipid signal characteristics are similar in both zones, the concentration of refractory plant waxes is higher in the homogeneous zones. This is further evidence that nonlaminated zones are not merely churned but also represent times of slower accumulation. The abundance of diatom fragments indicates that dissolution was more pronounced, but upwelling species are also rarer.

Until other types of analysis resolve problems of differential accumulation rates, we conclude, from the data, that nonlaminated zones record times when surface production of diatoms was lower because of reduced upwelling. One mechanism for this may involve a slightly lower salinity "lid," in concert with wet climate and glacial meltwater influx from the Colorado River or landward pluvials. Changing glacial-period wind patterns must also be evaluated. Nonlaminated sediments thus accumulate more slowly by the proportional lack of diatom population. Lower influx rates deplete less of the available oxygen in the water mass. Epifauna and infauna then further contribute to the destruction of available organic matter and delicate frustules by ingestion. There is insufficient evidence for an alternative explanation of increased terrigenous overprint on a relatively uniform diatom supply during glacial stages. In that case, oxygenated bottom waters would have to reflect stronger currents, overturn, and recharge from longer storm seasons.

The foregoing discussions are not intended as an exhaustive treatment of Site 480 , but rather to introduce the reader to problems raised by the raw data presented in the following chapters. There is a tremendous store of detailed climate-oceanographic information locked in Site 480 once the time keys are found.

\section{ACKNOWLEDGMENTS}

This paper is an expanded version of descriptive parts of the shipboard party's preliminary report (Schrader et al., 1980). We thank staff personnel of DSDP for technical assistance and preparation of figures. D. Ruegg typed an early version of the manuscript. We thank K. Pisciotto and P. Baumgartner for reviews and comments.

\section{REFERENCES}

Byrne, J., and Emery, K., 1960. Sediments of the Gulf of California. Geol. Soc. Am. Bull., 71:983-1010.

Calvert, S., 1964. Factors affecting distribution of laminated diatomaceous sediments in Gulf of California. In van Andel, Tj. H., and Shor, G. G. (Eds.), Marine Geology of the Gulf of California. Am. Assoc. Pet. Geol. Mem. 3:311-330.

1966. Origin of diatom-rich varved sediments from the Gulf of California. J. Geol., 76:546-565.

Revelle, R., 1950. Sedimentation and oceanography-survey of field observations. In Anderson, C. A., Durham, J. W., Shephard, F. P., Natland, M. L., and Revelle, R. (Eds.), The 1940 E.W. Scripps Cruise to the Gulf of California. Geol. Soc. Am. Mem. 43:1-6.

Roden, G. I., 1964. Oceanographic aspects of Gulf of California. In van Andel, Tj. H. and Shor, G. G. (Eds.), Marine Geology of the Gulf of California. Am. Assoc. Pet. Geol. Mem. 3:30-58.

Schrader, H. S., Kelts, K., Curray, J., Moore, D., Aguayo, E., Aubry, M. P., Einsele, G., Fornari, D., Gieskes, J., Guerrero, J., Kastner, M., Lyle, M., Matoba, Y., Molina-Cruz, A., Niemitz, J., Rueda, J., Saunders, A., Simoneit, B., and Vacquier, V., 1980. Laminated diatomaceous sediments from the Guaymas Basin Slope (central Gulf of California): 250,000-year climate record. Science, 207: 1207-1209.

Soutar, A., and Crill, P., 1977. Sedimentation and climatic patterns in the Santa Barbara Basin during the 19th and 20th centuries. Geol. Soc. Am. Bull., 88:1161-1172.

Zeitschel, B., 1969. Primary productivity in the Gulf of California. Mar. Biol., 3:201-207. 

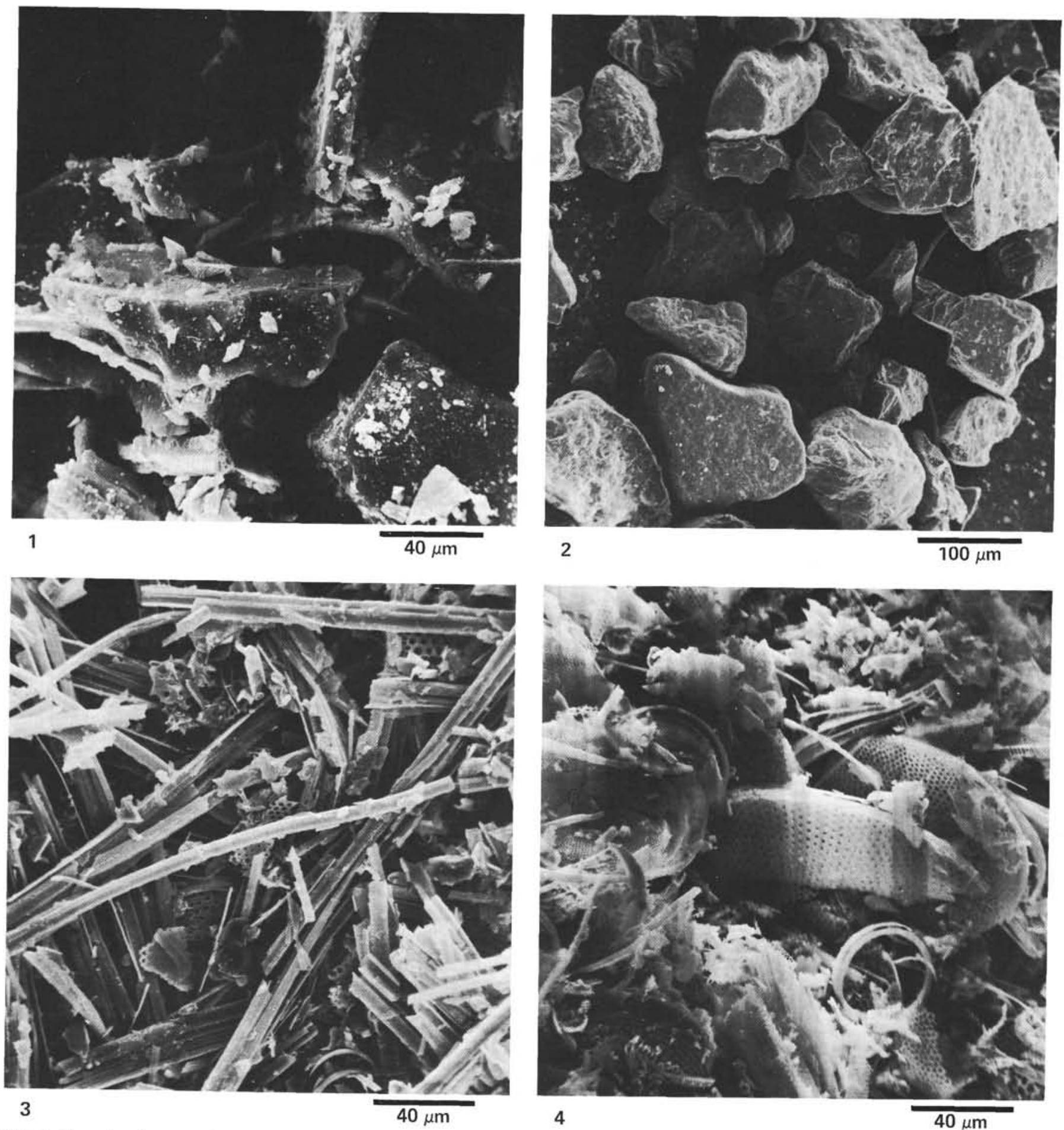

Plate 1. Scanning electron micrographs of Hole 480 sediments. 1. Ash layer, Sample $479-18-1,80 \mathrm{~cm}$, with etched, glassy shards. 2 . Sand grains,
Sample $480-20-1,10 \mathrm{~cm}$, species; 4. Dark laminae from couplet in Core 480-2; robust sand layer. 3. Light laminae from couplets in Core 480-2; fragile upwelling 